\title{
MARKET CONSISTENT PRICING OF INSURANCE PRODUCTS
}

\author{
BY
}

Semyon Malamud, Eugene Trubowitz and Mario V. Wüthrich*

\begin{abstract}
We present the first step in a program to develop a comprehensive, unified equilibrium theory of asset and liability pricing. We give a mathematical framework for pricing insurance products in a multiperiod financial market. This framework reflects classical economic principles (like utility maximization) and generates pricing algorithms for non-hedgeable insurance risks.
\end{abstract}

\section{KEYWORDS}

Market-consistent pricing, equilibrium theory, utility maximization, optimal consumption stream, insurance risk, non-hedgeable risk, idiosyncratic risk, indifference price, power utility, first order condition.

\section{INTRODUCTION}

This paper presents the first step in a program to develop a comprehensive, unified theory of equilibrium asset and liability pricing. We give a mathematical framework for pricing insurance products in a multiperiod financial market. This framework reflects classical economic principles and generates pricing algorithms for non-hedgeable insurance risks. These algorithms simultaneously price insurance products and maximize the utility of a (insurance company) shareholder's consumption stream. Thus, the objective of a market-consistent valuation of insurance liabilities is realized.

To endogenously price financial assets, one classically posits a utility maximization problem for the consumption stream of an agent and then imposes market clearing to define equilibria. There is a huge literature on the equilibrium pricing of financial instruments in complete markets (see, for example, Cochrane [3], Duffie [10], Merton [24], Ingersoll [20], Elliott-Kopp [11], TsanakasChristofides [32], Föllmer-Schied [13], Horst-Muller [19] and the reference therein). In particular, Horst-Muller [19] study an important problem of equilibrium pricing of insurance-related contracts (such as catastrophic bonds).

* ETH Zurich, Department of Mathematics, CH-8092 Zurich, Switzerland. 
If, in addition to trading financial assets (even those highly correlated with future claims such as in Horst-Muller [19]), the agent wants to directly sell insurance products (that he can not perfectly replicate with financial instruments) the market is necessarily incomplete. Concretely, the agent takes risks that are non-hedgeable, or in other words, the agent sells non-traded assets like insurance products.

The equilibria of incomplete markets are only poorly understood. The most common approaches to incomplete markets are to consider either a one-period model or the easier multiperiod utility maximization problem for the final wealth. We study here the more appropriate intertemporal wealth consumption in a multiperiod model. In Malamud-Trubowitz [22, 23], new methods are introduced for explicitly constructing optimal intertemporal consumption streams and obtaining explicit information about equilibria for general idiosyncratically incomplete markets.

An essential prerequisite for any attempt at market consistent pricing of insurance liabilities is an adequate notion of "optimal" premia streams. In this paper, we adopt the natural notion of a utility indifferent premia stream for compensating insurance claims. Suprisingly, it is possible to extend the method of Malamud-Trubowitz $[22,23]$ to explicitly construct static premia streams in a multiperiod environment for general intertemporal wealth consumption distributions. These premia streams are considered to be "fair" with respect to the chosen utility function. In the recent literature such a "fair" price is called an indifference price or a market-consistent price (see, for instance, Pelsser [28]). The notion of a fair price was first introduced by Hodges-Neuberger [18] (see, also, Davis [4] for notion of a fair price, based on the marginal value) and then extended by Barrieu-El Karoui [1] to the case of general risk measures and transaction costs. See, also, Delbaen et al. [7], Rouge-El Karoui [29], MusielaZariphopoulou [26, 27] or Henderson-Hobson [17] and references therein.

Note that the problem that we study here is, even though often being related to the classical problem of dividend optimization (see, e.g, de Finetti [5] and Dickson-Waters [9]), completely different. The classical de Finetti problem considers the maximization of present values of future dividends to shareholders until ruin. In that problem the insurance company is free to decide how much and when it wants to pay to the shareholders, moreover there are no financial markets! The problem we study here is completely different. Namely, the insurance company can use financial markets to reduce the risk by trading various financial assets. The maximization problem of the insurance company is not to find an optimal dividend process directly, but rather to find the optimal hedging strategy that reduces and reallocates the insurance risks through the financial markets in the way most preferable for the shareholders. The problem of finding optimal hedging strategies is crucial for modern insurance companies, especially after the appearance of insurance-related assets such as CAT-bonds (see, e.g., Horst-Muller [19], Muermann [25], Schmock [30]) that can be used to substantially reduce risk exposures and to mobilize risk capital/reinsurance.

The main mathematical point of this paper is that the optimal consumption and fair static premia streams are constructed simultaneously by exploiting a 
hidden inductive structure. We also implement the construction computationally.

We conclude that there is a vast literature devoted to optimal consumption streams in incomplete markets, see for instance Duffie [10]. However, the only existing results concern explicit solutions for very specific/special stochastic processes in continuous time using Hamilton-Jacobi-Bellman (HJB) equations. There is almost nothing known about the structure of optimal consumption streams in general incomplete markets. Only recently, Malamud-Trubowitz [22] discovered a large class of incomplete markets for which it is possible to construct the optimal consumption stream (and, consequently, the optimal hedging strategy) by an explicit, recursive procedure. In this paper we extend this construction and use it to determine the market consistent insurance premium for the class of incomplete markets, constructed in Malamud-Trubowitz [22]. We mention that a similar recursive structure has been discovered by Musiela-Zariphopoulou $[26,27]$ in a binomial model with an exponential utility for terminal wealth.

For us, the construction of static premia streams in the present paper is the jumping off point to a general theory of dynamic market consistent actuarial pricing. A general theory should include at least the following elements.

\section{Topics for future research: the next steps}

It looks feasible to:

- Complete the program for the special case of static premia by constructing market consistent equilibria and then deriving explicit pricing formulas. That is, writing the prices of financial assets and insurance claims explicitly in terms of risk aversion, insurance claims processes, endowment processes, etc.

- Introduce a conceptual framework for general dynamic (path-dependent) premia streams flexible enough to, among other essential features, allow insurance companies to adjust premia over time. Then, extend the method of this paper to construct path-dependent premia streams explicitly.

- Complete the general program for dynamic premia by constructing market consistent equilibria and derving explicit pricing formula.

- Enhance the role of the regulator. For the moment, the regulator excludes, by fiat, ruin and imposes investment constraints (borrowing constraints). Exploit our framework to define probability distortions, risk measures and regulatory requirements and use our market consistent equilibrium pricing to compute ruin probabilities.

\section{Organisation of this manuscript}

- In Section 2 we introduce the financial market model, the insurance model and consumption streams under expected utility considerations. Moreover, we derive first properties of the market consistent insurance premia. 
- In Section 3 we state existence and uniqueness of optimal consumption streams both, under the complete and the incomplete financial market assumption. This leads to different equations that are satisfied by optimal consumption streams.

- Section 4 is the heart of our framework which gives the inductive structure for the optimal consumption stream (see Theorem 4.3). This inductive structure gives the basic algorithm that is computationally implemented.

- In order to make market behaviour more realistic, we introduce borrowing constraints. These are constituted be the regulator. Under these constraints we derive the differential equation for the optimal consumption stream and its inductive structure. This is done in Section 5.

- Section 6 gives a (toy) example that demonstrates the computational implemention resulting in the static premia as a function of risk aversion.

- Finally, in the appendix we give all the proofs of the statements in Section 2 - Section 5 . The reason for putting the proofs to the appendix is to facilitate the readability of the paper. The reader should not worry about technical details when he reads this document for his first time. Indeed, we believe that the understanding of the basic economic properties in this model is as important as the technical details of the proofs of the statements.

\section{FinANCIAL AND INSURANCE MODEL}

\subsection{Financial market model}

Fix a probability space $(\Omega, \mathcal{B}, P)$. We consider $T+1$ time periods and the associated filtration $\mathcal{F}=\left(\mathcal{F}_{t}\right)_{t=0, \ldots, T}$ in the underlying $\sigma$-algebra $\mathcal{B}$. This filtration $\mathcal{F}$ contains all information about financial events (financial filtration).

ASSUMPTION 1. There is a fixed financial market $(\mathcal{M}, \mathcal{F})$ trading in L financial assets $A_{1}, \ldots, A_{L}$ adapted to $\mathcal{F}$. There are no other financial assets.

The financial assets $A_{j}, j=1, \ldots, L$, are characterized by two positive processes, a price process

$$
\mathbf{q}_{j}=\left(q_{j, t}: t=0, \ldots, T\right),
$$

and a dividend payout process

$$
\mathbf{D}_{j}=\left(D_{j, t}: t=0, \ldots, T\right) .
$$

Observe that the zero coupon bond with maturity $m \in\{1, \ldots, T\}$ is modelled by the dividend process $D_{t}=1_{\{t=m\}}$ and with price process satisfying $q_{t}=0$ for $t \geq m$. 
An $\mathcal{F}$ adapted portfolio trading strategy is an $L$ dimensional, $\mathcal{F}$ adapted process

$$
\mathbf{x}=\left(\mathbf{x}_{1}, \ldots, \mathbf{x}_{L}\right), \quad \text { with } \quad \mathbf{x}_{j}=\left(x_{j, 0}, \ldots, x_{j, T-1}, 0\right)
$$

The random variable $x_{j, t}$ counts the number of shares of asset $A_{j}$ held within the time interval $(t, t+1)$. The last component 0 formalizes the convention that no investments are made at the terminal time $T$.

DEFINITION 2.1. The dividend process $\mathbf{D}_{\mathbf{x}}$ generated by the portfolio trading strategy $\mathbf{x}$ is for $t=1, \ldots, T$ given by

$$
D_{\mathbf{x}, t}=\sum_{j=1}^{L}\left(D_{j, t}+q_{j, t}\right) x_{j, t-1}-\sum_{j=1}^{L} q_{j, t} x_{j, t},
$$

and the initial investment at time $t=0$ is

$$
D_{\mathbf{x}, 0}=-\sum_{j=1}^{L} q_{j, 0} x_{j, 0} .
$$

$D_{\mathbf{x}, t}$ formalizes the payoff of the investments made at time $t-1$, minus the new investments made at time $t$.

Note that we use $x_{j, t}$ to denote the portfolio holding at time $t+$ and therefore $x_{j, t}$ is $\mathcal{F}_{t}$-measurable. To avoid confusion, we note that often in financial literature $x_{j, t}$ is used to denote the number of shares bought at time $t-1$ and therefore $x_{j, t}$ in that literature is $\mathcal{F}_{t-1}$-measurable (previsible), that is, in our convention the time index is shifted by one step.

\subsection{State price densities}

A powerful concept of modern asset pricing is that of state price densities. Duffie [10] emphasizes their importance in modern finance. In actuarial mathematics state price densities are also called deflators (see Duffie [10] and Bühlmann et al. [2]).

Definition 2.2. A process $\mathbf{M}=\left(M_{t}\right)_{t=0, \ldots, T}$ adapted to the filtration $\mathcal{F}$ is a state price density process of the market $(\mathcal{M}, \mathcal{F})$ when

$$
M_{t} q_{j, t}=E\left[M_{t+1}\left(q_{j, t+1}+D_{j, t+1}\right) \mid \mathcal{F}_{t}\right]
$$

for all $j=1, \ldots, L$ and $t=0, \ldots, T-1$.

The following lemma is an immediate consequence of the definition. 
LEMMA 2.3. A process $\mathbf{M}$ is a state price density process if and only if

$$
E\left[\sum_{t=0}^{T} M_{t} D_{\mathbf{x}, t}\right]=0
$$

for all portfolio trading strategies $\mathbf{x}$. Equivalently, a process $\mathbf{Y}$ is a dividend process of a portfolio strategy if and only if

$$
E\left[\sum_{t=0}^{T} M_{t} Y_{t}\right]=0
$$

for all state price density processes $\mathbf{M}$.

Proof. The proof is provided in Lemma B.1, (a) $\Leftrightarrow$ (c).

The above lemma says that state price densities are natural dual objects to asset prices with respect to the standard inner product. They allow for formulating important questions in an elegant form. For example, it is well known that (under some natural technical conditions) the absence of arbitrage in the market $(\mathcal{M}, \mathcal{F})$ is equivalent to the existence of a positive state price density process (see, for example, Delbaen-Schachermayer [8], Föllmer-Schied [13], Duffie [10], Wüthrich et al. [33]). Another important consequence of (2.6) is the identity

$$
q_{j, t}=E\left[\sum_{\tau=t}^{T} \frac{M_{\tau}}{M_{t}} D_{j, \tau} \mid \mathcal{F}_{t}\right] .
$$

That is, dividend process and the state price density determine the asset price process.

If, in addition, the market $(\mathcal{M}, \mathcal{F})$ is complete, the state price density process is unique (see, for example, Föllmer-Schied [13] and Duffie [10]). In the sequel we make the following assumption.

ASSUMPTION 2. We assume that the financial market $(\mathcal{M}, \mathcal{F})$ is arbitrage-free and complete, i.e. there exists an a.s. unique positive state price density process $\mathbf{M}=\left(M_{t}\right)_{t=0, \ldots, T}$ normalized by $M_{0}=1$, that satisfies Definition 2.2.

\subsection{Expected utility theory and optimal consumption}

Our financial company is endowed with an $\mathcal{F}$ adapted exogenous stream of income $\mathbf{w}=\left(w_{t}\right)_{t=0, \ldots, T}$ referred to as the endowment process. Now, being a financial company, it invests the endowments into assets and trades them in the financial market. That is, the company chooses a portfolio trading strategy $\mathbf{x}$, and consumes the resulting payoffs. Its consumption stream $\mathbf{c}=\mathbf{c}(\mathbf{w}, \mathbf{x})=$ $\mathbf{c}(0,0, \mathbf{w}, \mathbf{x})=\left(c_{t}\right)_{t=0, \ldots, T}$ for the portfolio strategy $\mathbf{x}$ is given by

$$
\mathbf{c}=\mathbf{w}+\mathbf{D}_{\mathbf{x}}
$$


This means, the consumption stream $\mathbf{c}$ is given by the endowment process $\mathbf{w}$ and the resulting dividend process $\mathbf{D}_{\mathbf{x}}$. Note that, since $x_{j, T}=0$, the last consumption corresponds to the final wealth, i.e., we consider intertemporal wealth consumption plus final wealth at time $T$.

In applications to real life, $\mathbf{c}$ corresponds to the dividend process that the company pays to the shareholders. But, in contrast to the classical de Finetti problem [5], the company trades on the financial market to achieve the optimal portfolio investment strategy that hedges the shareholders risks in the best possible way. This we are just going to explain in the sequel.

There are many different reasons for which the financial company trades in the market, for example, various types of hedging and speculative strategies. But, after all, the decisions are determined by maximizing profits under investment constraints. We assume that the shareholder is a rational economic agent, maximizing expected, discounted intertemporal utility function: For $\gamma>0, \rho>0$ and $\mathbf{c}>0$ we define

$$
U(\mathbf{c})=E\left[\sum_{t=0}^{T} u_{\rho, \gamma}\left(t, c_{t}\right)\right]=E\left[\sum_{t=0}^{T} e^{-\rho t} u\left(c_{t}\right)\right]=E\left[\sum_{t=0}^{T} e^{-\rho t} \frac{c_{t}^{1-\gamma}}{1-\gamma}\right] .
$$

$\gamma$ is the risk aversion of the economic agent and $\rho$ stands for his impatience in time.

\section{REMARKS 2.4.}

The benchmark case of power utility function $u(c)=c^{1-\gamma} /(1-\gamma)$ has become standard in the financial literature (see, for example, Cochrane [3], for an actuarial example, see de Jong [6]). This is the constant relative risk aversion (CRRA) utility. It is often difficult to work with this power utility function, and hence, even though widely accepted from an economic viewpoint, many people prefer working with the exponential utility function (constant absolute risk aversion (CARA), see, e.g., Delbaen et al. [7], Rouge-El Karoui [29], Henderson [15, 16], Musiela-Zariphopoulou [26, 27] or Pelsser [28]). Hence, even though it is now a common belief that the exponential utility does not properly describe investor's behavior, it is still very popular because of its nice multiplicative properties (for example, this multiplicative structure is the reason why the construction of Musiela-Zariphopoulou [26, 27] works).

Working with CRRA utility requires more advanced methods. In MalamudTrubowitz $[22,23]$ techniques are presented that work for any utility function.

We also mention that none of the preceding papers on this topic (see, e.g., [7], [15], [16], [26], [27], [28], [29]) incorporates intertemporal consumption. We emphasize that it is crucial to incorporate intertemporal consumption into the company's decision making processes. After all, the company has to close its books every year and pay yearly dividends to the shareholders, i.e., the natural view is based on intertemporal utility considerations. 
DEFINITION 2.5. The utility maximization problem for an agent with endowment process $\mathbf{w}=\left(w_{t}\right)_{t=0, \ldots, T}$ is to maximize the utility achievable for the consumption stream $\mathbf{c}(\mathbf{w})=\mathbf{c}(0,0, \mathbf{w}, \mathbf{x})=\mathbf{w}+\mathbf{D}_{\mathbf{x}}>0$; i.e., determine

$$
U^{\max }(\mathbf{w}) \stackrel{\text { def }}{=} \max _{\mathbf{x}} U(\mathbf{c}(0,0, \mathbf{w}, \mathbf{x}))=\max _{\mathbf{x}} U\left(\mathbf{w}+\mathbf{D}_{\mathbf{x}}\right) .
$$

Below we make Technical Assumption 1, which implies that the existence of the maximum in (2.11) is trivial (see, for example, He-Pearsson [14]). A simple consequence of the definition is the following lemma.

LEMMA 2.6. $U^{\max }(\mathbf{w})$ is a strictly increasing and concave function. In a complete market $(\mathcal{M}, \mathcal{F})$, the optimal consumption stream and, consequently, the maximal utility achievable $U^{\max }(\mathbf{w})$ only depends on the intertemporal wealth $W$ of the endowment process $\mathbf{w}$ given by

$$
W=E\left[\sum_{t=0}^{T} w_{t} M_{t}\right]
$$

Here, $\mathbf{M}=\left(M_{t}\right)$, is the unique state price density process in the complete market.

\subsection{Insurance market model}

It is clear that most insurance claims are not completely correlated with the asset market. Therefore, it is not possible to completely replicate insurance claims with financial instruments, and hence, insurance claims are not adapted to the financial filtration $\mathcal{F}$.

For modelling the insurance claims payment stream $\mathbf{Y}=\left(0, Y_{1}, \ldots, Y_{T}\right)$ with $Y_{t} \geq 0, t=1, \ldots, T$, we introduce a second, larger filtration $\mathcal{G}=\left(\mathcal{G}_{t}\right)_{t=0, \ldots, T}$ in the underlying $\sigma$-algebra $\mathcal{B}$. This filtration contains all financial information and all insurance technical information. That is, we assume for all $t=0, \ldots, T$ that

$$
\mathcal{F}_{t} \subset \mathcal{G}_{t} \text {, for } t=1, \ldots, T \text {, and } \mathcal{F}_{0}=\mathcal{G}_{0}=\{\emptyset, \Omega\} .
$$

We make the following

Technical assumption 1. The $\sigma$-algebra $\mathcal{G}_{t}$ is finite for all $t=0, \ldots, T$.

Technical Assumption 1 allows for formulating results and proofs in a simple way that directly display the essential economic points. It would be possible to drop Technical Assumption 1, but then the analysis becomes much more technical and sophisticated, for example, without Technical Assumption 1 the solution to the utility maximization problem may fail to satisfy the first order conditions (given below). Therefore we omit this extension here because already under 
Technical Assumption 1 we can highlight the main economic features. Note that the finiteness of $\mathcal{G}_{t}$ implies the finiteness of $\mathcal{F}_{t}$.

We introduce incompleteness by the enlargement of the financial filtration $\mathcal{F}$ (this can be viewed as adding individual information to the financial market information which gives idiosyncratic risk). For general incomplete markets, it is impossible to make any economically interesting statements about the optimal consumption stream (see, e.g., Duffie [10]). It is the special elegant structure of the market incompleteness, directly encoded in the two filtrations $\mathcal{G}$ and $\mathcal{F}$, that allows to use the methods of Malamud-Trubowitz [22] and perform our analysis. Note that, because of the market incompleteness, there are infinitely many state price density processes adapted to the larger filtration $\mathcal{G}$. But there is one special, unique, aggregate state price density process $\mathbf{M}$ adapted to $\mathcal{F}$.

\subsection{Insurance premia}

The insurance company, facing insurance claims flow $\mathbf{Y}$, of course, requires compensation in the form of a premia flow $\Pi=\left(\Pi_{t}\right)_{t=0, \ldots, T}$.

The modified consumption stream $\mathbf{c}=\mathbf{c}(\Pi, \mathbf{Y}, \mathbf{w}, \mathbf{x})=\left(c_{t}\right)_{t=0, \ldots, T}$ under insurance is given by

$$
\mathbf{c}=\mathbf{w}+\Pi+\mathbf{D}_{\mathbf{x}}-\mathbf{Y}=(\mathbf{w}+\Pi-\mathbf{Y})+\mathbf{D}_{\mathbf{x}} .
$$

Our aim is to study different types of premia flows $\Pi$ and to determine premia flows that are considered to be "fair". Equality (2.14) indicates that adding an insurance process simply means modifying the endowment process

$$
\mathbf{w} \mapsto \tilde{\mathbf{w}}=\mathbf{w}+\Pi-\mathbf{Y},
$$

which is, for $\mathcal{G}$ adapted claims $\mathbf{Y}$ and premia flows $\Pi$, now a $\mathcal{G}$ adapted endowment process $\tilde{\mathbf{w}}$. On the same hand, we will choose $\mathcal{G}$ adapted investment strategies $\mathbf{x}$ whereas, of course, the price and dividend processes of the assets $A_{j}$ remain $\mathcal{F}$ adapted.

\subsubsection{Static insurance premium}

The static premium corresponds to the premia flow $\Pi_{0}=\left(\pi_{0}, 0, \ldots, 0\right)$, with $\pi_{0}$ being $G_{0}$-measurable. That is, there is one single premium installment at the beginning of the insurance period. An example is selling/buying an insurance run-off portfolio with a single payment.

Since our insurance company has access to the financial market, it of course uses it to hedge a part of the insurance risks and develops an optimal hedging strategy $\mathbf{x}$. Note that until now all the shocks that the financial company was exposed to were adapted to the aggregate filtration $\mathcal{F}$. Consequently, the portfolio strategy $\mathbf{x}$, was also adapted to the aggregate filtration $\mathcal{F}$. Now, being 
exposed to a $\mathcal{G}$ adapted claims payment stream $\mathbf{Y}$, the company will of course use a $\mathcal{G}$ adapted portfolio strategy $\mathbf{x}$ to hedge the insurance risks. If the insurance company chooses a $\mathcal{G}$ adapted hedging strategy $\mathbf{x}$, the static premium $\Pi_{0}$ generates the consumption stream $\mathbf{c}\left(\Pi_{0}, \mathbf{Y}, \mathbf{w}, \mathbf{x}\right)$ with

$$
\begin{aligned}
& c_{0}=w_{0}+D_{\mathbf{x}, 0}+\pi_{0}, \\
& c_{t}=w_{t}+\mathrm{D}_{\mathbf{x}, t}-Y_{t}, \quad \text { for } t \geq 1 .
\end{aligned}
$$

In this paper we do not consider the possibility of ruin. Thus, the company should require a premium that is at least sufficient to generate a positive consumption stream (2.16)(2.17). In particular, the CRRA utility function is only defined for positive consumption $c_{t}$ and the utility is maximized over the budget set

$$
B\left(\mathbf{w}-\mathbf{Y}+\Pi_{0}\right)=\left\{\mathbf{c} \text { fulfills (2.16)-(2.17) with } c_{t}>0 \text { for } t=0, \ldots, T\right\},
$$

and optimizing over the investment strategies $\mathbf{x}$

$$
U^{\max }\left(\mathbf{w}+\Pi_{0}-\mathbf{Y}\right)=\max _{\mathbf{C} \in B\left(\mathbf{w}-\mathbf{Y}+\Pi_{0}\right)} U(\mathbf{c}) .
$$

Thus, even to be able to define the value function we need that the budget set be non-empty. For this purpose we will need the following definition.

DEFINITION 2.7. Let $\mathbf{Z}=\left(Z_{t}\right)_{t=1, \ldots, T}$ be a $\mathcal{G}$ adapted process. The upper hedging price $\mathbf{Z}^{u}$ is the minimal number such that there exists a portfolio strategy $\mathbf{x}$ with $\mathbf{Z}^{u}+D_{\mathbf{x}, 0}=0$ and $D_{\mathbf{x}, t}-Z_{t} \geq 0$ for all $t=1, \ldots, T$. Such a portfolio strategy is called super-replicating. The process $\left(Z_{t}\right)$ is called replicable if there exists a portfolio strategy $\mathbf{x}$ such that $D_{\mathbf{x}, t}=Z_{t}$ for all $t=1, \ldots, T$.

The upper hedging price is the minimal price for which one can buy a portfolio strategy that hedges all possible losses from $Z_{t}$ from above. If the market is complete, it is possible to find a replicating portfolio strategy such that $D_{\mathbf{x}, t}=Y_{t}$ for $t=1, \ldots, T$, that is, any $\mathcal{F}$-adapted process $\mathbf{Y}$ is replicable in the complete market $(\mathcal{M}, \mathcal{F})$. But for incomplete markets, we can only talk about super-replicating. The following lemma contains some well-known properties of the upper hedging price. See, e.g., Schachermeyer [31], Theorem 2.11.

LEMMA 2.8. The upper hedging price for the $\mathcal{G}$ adapted process $\mathbf{Z}$ is given by

$$
\mathbf{Z}^{u}=\max E\left[\sum_{t=1}^{T} R_{t} Z_{t}\right]
$$

where the maximum is taken over all positive state price density processes $\mathbf{R}$ of $(\mathcal{M}, \mathcal{G})$ with $R_{0}=1$. If the process $\mathbf{w}$ is replicable then 


$$
(\mathbf{Z}-\mathbf{w})^{u}=\mathbf{Z}^{u}-E\left[\sum_{t=1}^{T} M_{t} w_{t}\right] .
$$

Recall that

$$
W=E\left[\sum_{t=0}^{T} M_{t} w_{t}\right]
$$

is the intertemporal wealth of the endowment process $\mathbf{w}$. We have:

Lemma 2.9. The maximal expected utility $U^{\max }\left(\mathbf{w}+\Pi_{0}-\mathbf{Y}\right)$ is defined if and only if

$$
\pi_{0}>\mathbf{Y}^{u}-W
$$

If $\gamma>1$ then we have

$$
\begin{aligned}
\infty= & \lim _{\pi_{0} \rightarrow \mathbf{Y}^{u}-W}(1-\gamma) U^{\max }\left(\mathbf{w}+\Pi_{0}-\mathbf{Y}\right) \\
& >\lim _{\pi_{0} \rightarrow \infty}(1-\gamma) U^{\max }\left(\mathbf{w}+\Pi_{0}-\mathbf{Y}\right)=0 .
\end{aligned}
$$

If $\gamma<1$ and $\mathbf{Y}$ is not replicable then we have

$$
\begin{aligned}
0< & \lim _{\pi_{0} \rightarrow \mathbf{Y}^{u}-W} U^{\max }\left(\mathbf{w}+\Pi_{0}-\mathbf{Y}\right) \\
& <\lim _{\pi_{0} \rightarrow \infty} U^{\max }\left(\mathbf{w}+\Pi_{0}-\mathbf{Y}\right)=\infty .
\end{aligned}
$$

The special recursive structure of our market incompleteness allows to expliticely calculate the upper hedging price, see Lemma 3.5, below.

Now we are ready to define the market consistent premia.

DefinITION 2.10. We fix a G adapted endowment process $\mathbf{w}$. A $\mathcal{G}_{0}$-measurable static premium $\Pi_{0}$ is called market consistent for the claims payment stream $\mathbf{Y}$ if

$$
U^{\max }\left(\mathbf{w}+\Pi_{0}-\mathbf{Y}\right)=U^{\max }(\mathbf{w}) .
$$

Formula (2.26) reflects the natural actuarial "fair price" principle, see, e.g., Pelsser [28], Hodges-Neuberger [18], also referred to as the utility indifference valuation: an insurance company with utility function $U$ is indifferent between selling this insurance contract and not selling it. The only tricky thing is that it is not the utility that stands there, but the maximal utility achievable by optimal trading! Because the insurance company is able to reduce the risk exposure by trading on the financial market, the fair price required is below the price, it would require if there was no financial market. 
THEOREM 2.11. The following holds true:

The market consistent static premium $\Pi_{0}$ exists if and only if $\gamma<1$ and

$$
\lim _{\pi_{0} \rightarrow \mathbf{Y}^{u}-W} U^{\max }\left(\mathbf{w}+\Pi_{0}-\mathbf{Y}\right)<U^{\max }(\mathbf{w}) .
$$

or $\gamma>1$.

If $\Pi_{0}$ exists, it is a unique and convex function of the insurance claims $\mathbf{Y}$ and satisfies

$$
\mathbf{Y}^{u}-W<\pi_{0}<\mathbf{Y}^{u}
$$

Moreover, if $\mathbf{Y}^{(1)} \leq \mathbf{Y}^{(2)}$ a.s., then $\pi_{0}\left(\mathbf{Y}^{(1)}\right) \leq \pi_{0}\left(\mathbf{Y}^{(2)}\right)$.

There is an important difference between the cases $\gamma>1$ and $\gamma<1$. In the case $\gamma>1$ the utility is always negative, due to the factor $(1-\gamma)^{-1}$. Moreover, it converges to $-\infty$ when the wealth goes to zero. When $\gamma<1$ the utility is always positive. Furthermore, when the market is complete, consumption in all states converges to zero when the wealth goes to zero.

In incomplete markets this picture can not happen: consumption can not go to zero in all states only if the claims payment stream is perfectly hedgeable. Otherwise, there are always states in which the claims can not be replicated. Since we do not allow for ruin, consumption in those states is always positive. Consequently, the utility always stays strictly positive, even if the wealth is so small that it only allows for avoiding ruin. If a company with a small initial wealth sells an insurance contract, then it requires a sufficient amount of premium in order to avoid ruin, the new utility becomes much larger than the initial one. Thus, there is no indifference price.

\subsubsection{Constant annual insurance premium}

The constant annual insurance premium case corresponds to the premia flow $\Pi_{a n n}=\left(0, \pi_{a n n}, \ldots, \pi_{a n n}\right)$, with $\pi_{a n n}$ is $\mathcal{G}_{0}$-measurable. This means that we consider multiperiod contracts which have a fixed annual premium $\pi_{a n n}$ over the whole period of the contract. It is paid in arrears, using zero coupon bonds however, this can easily be understood as a premium paid in advance.

The constant insurance premium case generates the following consumption stream $\mathbf{c}\left(\Pi_{a n n}, \mathbf{Y}, \mathbf{w}, \mathbf{x}\right)$ given by

$$
\begin{aligned}
& c_{0}=w_{0}+D_{\mathbf{x}, 0}, \\
& c_{t}=w_{t}+D_{\mathbf{x}, t}+\pi_{a n n}-Y_{t}, \quad \text { for } t \geq 1 .
\end{aligned}
$$


DeFINITION 2.12. Fix a $\mathcal{G}$ adapted endowment process $\mathbf{w}$. The $\mathcal{G}_{0}$-measurable constant annual premium $\Pi_{\text {ann }}$ is called market consistent for the claims payment stream $\mathbf{Y}$ if

$$
U^{\max }\left(\mathbf{w}+\Pi_{a n n}-\mathbf{Y}\right)=U^{\max }(\mathbf{w}) .
$$

Because in complete markets we can perfectly transfer wealth between different time periods, using risk free bonds, the following is true.

Proposition 2.13. Assume that the financial market $(\mathcal{M}, \mathcal{F})$ is complete, then

$$
\pi_{0}=\pi_{a n n} \sum_{t=1}^{T} E\left[M_{t}\right]
$$

The deeper reason for Proposition 2.13 to hold true is that we can borrow future premia from third party until it is due. That is, we do not care about the time points when the premia come in. Of course, this is not a realistic situation. In practice, there are usually constraints on borrowing money from third party (for example imposed by the regulator). We study such borrowing constraints in Section 5.

Note that, if $\mathbf{Y}$ is an $\mathcal{F}$ adapted cash flow, then, by Assumption 2, the price of an asset with dividend process $\mathbf{Y}$ is given by

$$
y_{0}=E\left[\sum_{t=1}^{T} M_{t} Y_{t}\right] \text {. }
$$

Therefore, the premia flow $\Pi$ is "fair" if and only if the discounted value is

$$
y_{0}=E\left[\sum_{t=1}^{T} M_{t} \Pi_{t}\right] .
$$

Now, when $\mathbf{Y}$, is $\mathcal{G}$ adapted, it can not be priced by arbitrage arguments and the quantity (2.33) is no longer considered as the unique "fair" price. (2.33) can still be viewed as the riskfree "fair" price, i.e., the market consistent price that a risk neutral insurance company charges. But, when the company is risk averse $(\gamma>0)$ then the company requires an additional risk premium. Namely, the following is true.

LEMma 2.14. For any $\mathcal{G}$ adapted premia flow $\Pi$ with

$$
U^{\max }(\mathbf{w}+\Pi-\mathbf{Y}) \geq U^{\max }(\mathbf{w}),
$$

we have 


$$
E\left[\sum_{t=0}^{T} \Pi_{t} M_{t}\right] \geq E\left[\sum_{t=0}^{T} Y_{t} M_{t}\right] .
$$

Lemma 2.14 expresses that under risk aversion the expected insurance premium is larger than the expected discounted value of the claim Y. For example, for the static premium $\pi_{0}$ Lemma 2.14 gives the inequality

$$
\pi_{0} \geq E\left[\sum_{t=0}^{T} Y_{t} M_{t}\right]=y_{0} .
$$

The difference between the left and the right hand sides is the risk premium (risk loading) for the insurance contract that the insurance company charges. This risk premium depends on the size of risk aversion $\gamma$ and all other economically important parameters.

\subsubsection{Market consistency}

Muermann [25] shows that the price of an insurance contract must be related to the prices of existing assets to exclude arbitrage opportunities. Namely, he studies the problem of pricing insurance contracts insuring against catastrophic events. Muermann [25] obtains relations between the price of such an insurance contract and the price of a CAT-bond that exclude arbitrage opportunities (see, also, Hodges-Neuberger [19] for an equilibrium model of CAT-bond pricing).

In this paper we consider a different problem. We have a particular insurance company, characterized by its utility function and endowment. The company sells insurance contracts on any $\mathcal{G}$ adapted claims payment stream $\mathbf{Y}=\left(Y_{t}\right)_{t}$ and has a premium rule $\pi_{0}(\mathbf{Y})$ to price such contracts. Note that we only consider insurance contracts sold at time zero, but the analysis extends to the dynamic case. Market (or, in the language of Muermann [25], actuarial) consistency means that there are no arbitrage opportunities in the joint financial and insurance market. An arbitrage opportunity exists if it is possible to enter an insurance contract at time zero and find a trading strategy such so that together they generate positive profits with zero initial investment.

LEMmA 2.15. Suppose that the premium rule $\pi_{0}(\cdot)$ satisfies the inequality

$$
\pi_{0}(\mathbf{Y}) \geq E\left[\sum_{t=1}^{T} M_{t} Y_{t}\right]
$$

for any claims payment stream $\mathbf{Y}$. Then, the insurance and financial markets are jointly arbitrage free.

The following definition for market consistency is based on arbitrage-freeness. 
DeFINITION 2.16. A premium rule $\pi_{0}(\cdot)$ is market consistent if

(1) for any stream $\mathbf{Y}=\left(Y_{t}\right)$,

$$
\mathbf{Y}^{u}>\pi_{0}(\mathbf{Y}) \geq E\left[\sum_{t=1}^{T} M_{t} Y_{t}\right]
$$

and equality takes place if and only if $\mathbf{Y}$ is replicable;

(2) for any replicable stream $\mathbf{Z}$ and any other stream $\mathbf{Y}$ we have

$$
\pi_{0}(\mathbf{Y}+\mathbf{Z})=\pi_{0}(\mathbf{Y})+\pi_{0}(\mathbf{Z})
$$

Definition 2.16 has the following economic meaning. Item (1) guarantees (by Lemma 2.15) that there is no arbitrage. Economically this means that the insurance company only insures the unhedgeable risks contained in $\mathbf{Y}$ if one pays a higher price than the "fair" of these risks (discounted expected value (2.33)). The difference is the safety loading, that depends on various parameters such as the risk aversion of the shareholders, capital of the insurance company, solvency constraints, etc. Furthermore, the upper bound (upper hedging price) also tells us that it is profitable for an agent to enter the insurance contract, because it is more expensive for him to hedge the claims stream by directly trading in the financial market.

Item (2) means that the insurance company lives in a "perfect agreement" with the financial market and only charges the safety loadings for the unhedgeable part of the claims. The part of the claims that can be hedged is priced in a market consistent way, so that the insured is indifferent in whether to put this part of risks into the insurance company or to go to the financial market and hedge the financial risk himself.

Note that the utility indifference pricing rule for the static premium defined in Definition 2.10 satisfies this definition of market consistency (Lemma 2.14, Theorem 2.11 and the fact that $(\mathcal{M}, \mathcal{F})$ is complete) and is therefore called market consistent.

\section{OPTIMAL CONSUMPTION STREAMS}

\subsection{Complete financial market}

Recall that under Assumption 2 every $\mathcal{F}$ adapted financial process $\mathbf{D}=\left(D_{1}, \ldots\right.$, $D_{T}$ ) can be replicated by an $\mathcal{F}$ adapted portfolio trading strategy $\mathbf{x}$ such that for all $t=1, \ldots, T$

$$
D_{\mathbf{x}, t}=D_{t}
$$


THEOREM 3.1. Assume we have an $\mathcal{F}$ adapted endowment process $\mathbf{w}$ in the complete financial market $(\mathfrak{M}, \mathcal{F})$ such that its intertemporal value $W$ is positive. Then, there exists a unique $\mathcal{F}$ adapted consumption stream $\mathbf{c}(0,0, \mathbf{w}, \mathbf{x})$ that maximizes (2.10) according to (2.11). It is determined through the first order conditions

$$
\mathrm{cm}_{t} \stackrel{\text { def }}{=} c_{t}=c_{0} e^{-\rho t / \gamma} M_{t}^{-1 / \gamma} .
$$

Hence the law of optimal consumption streams can explicitly be calculated. It depends on the law of the state price density $\mathbf{M}$ only.

\subsection{Insurance market model and incompleteness}

Suppose $Y \in L^{2}(\Omega, \mathcal{B}, P)$ is an $\mathcal{F}_{t+1}$-measurable random variable. By construction $E\left[Y \mid \mathcal{F}_{t}\right]$ is the first component of $Y$ in the orthogonal decomposition

$$
L_{2}(\Omega, \mathcal{B})=L_{2}\left(\Omega, \mathcal{F}_{t}\right) \oplus L_{2}\left(\Omega, \mathcal{F}_{t}\right)^{\perp}
$$

Intuitively, it is the part of $Y$ that "one can know" at time $t$. We assume that knowledge of insurance events at time $t$ does not give us any information about financial events at time $t+1$.

Assumption 3. For each $t=0, \ldots, T-1$, and for every integrable random variable $Y$, measurable with respect to $\mathcal{F}_{t+1}$, we assume

$$
E\left[Y \mid \mathcal{F}_{t}\right]=E\left[Y \mid \mathcal{G}_{t}\right]
$$

REMARKS 3.2.

- Assumption 3 implies that for any random variable $Y$ measurable with respect to $\mathcal{F}_{t+\tau}, \tau \geq 1$,

$$
E\left[Y \mid \mathcal{F}_{t}\right]=E\left[Y \mid \mathcal{G}_{t}\right]
$$

- Of course, we may question Assumption 3. In practice, insurance shocks at time $t$ may have an influence on financial market events at time $t+1$. Assumption 3 simply means that this effect of insurance shocks is already contained in $\mathcal{F}$.

Lemma 3.3. Under Assumptions 1-3 M is also a state price density with respect to $\mathcal{G}_{t}$. That is,

$$
M_{t} q_{j, t}=\left[M_{t+1}\left(q_{j, t+1}+D_{j, t+1}\right) \mid \mathcal{G}_{t}\right]
$$

for all $j=1, \ldots, L$ and all $t=0, \ldots, T-1$. In particular, the market $(\mathcal{M}, \mathcal{G})$ is arbitrage free. 
The market $(\mathcal{M}, \mathcal{G})$ is obviously incomplete. There are infinitely many state price density processes satisfying (3.6). Below we present analysis of this special structure of incompleteness following Malamud-Trubowitz [22].

\subsection{Optimal consumption streams in incomplete markets}

Given a $\mathcal{G}$ adapted portfolio trading strategy $\mathbf{x}$ (recall that the price and dividend processes of all assets are $\mathcal{F}$ adapted), we define the financial wealth $X_{t}$ of the investments at time $t$ by

$$
X_{t}=\sum_{j=1}^{L}\left(D_{j, t}+q_{j, t}\right) x_{j, t-1} .
$$

We define $\mathcal{H}_{0}=\mathcal{F}_{0}$ and for $t=1, \ldots, T$

$$
\mathcal{H}_{t} \text { the } \sigma \text {-algebra generated by } \mathcal{F}_{t} \text { and } \mathcal{G}_{t-1} \text {. }
$$

Lemma 3.4 below gives an economic interpretation to the filtration $\mathcal{H}_{t}$ : it is the hedgeable filtration. Namely, any $\mathcal{H}_{t}$-measurable payoff can be replicated by a $\mathcal{G}_{t-1}$-measurable investment at time $t-1$. The reason is, of course, the completeness of the market $(\mathcal{M}, \mathcal{F})$. Knowing the insurance shocks at time $t-1$, we can perfectly hedge any claim that depends on these shocks and the aggregate shocks in the $\sigma$-algebra $\mathcal{F}_{t}$.

Lemma 3.4. The financial wealth $X_{t}$ is $\mathcal{H}_{t}$-measurable, and for any $\mathcal{H}_{t}$ adapted process $X_{t}$ there exists a $\mathcal{G}$ adapted portfolio strategy $\mathbf{x}$ having $X_{t}$ as the financial wealth process. The corresponding dividend process satisfies

$$
\begin{aligned}
D_{\mathbf{x}, t} & =\sum_{j=1}^{L}\left(D_{j, t}+q_{j, t}\right) x_{j, t-1}-\sum_{j=1}^{L} q_{j, t} x_{j, t} \\
& =X_{t}-E\left[\frac{X_{t+1} M_{t+1}}{M_{t}} \mid \mathcal{G}_{t}\right] .
\end{aligned}
$$

As mentioned above Lemma 3.4 is crucial, because it gives us the understanding of the set of replicable streams. In particular, we are now able to calculate the upper hedging price explicitly.

Lemma 3.5. Let $Y_{T+1}^{\text {sup }}=0$ and then define inductively for $t \leq T$

$$
Y_{t}^{\text {sup }}=\operatorname{esssup}\left[Y_{t}+M_{t}^{-1} E\left[Y_{t+1}^{\text {sup }} M_{t+1} \mid \mathcal{G}_{t}\right] \mid \mathcal{H}_{t}\right] .
$$

Then, $Y_{0}^{\text {sup }}=\mathbf{Y}^{u}$ is the upper hedging price. 
Consider $\mathcal{G}$ adapted endowment processes $\mathbf{w}$ and investment strategies $\mathbf{x}$ with their $\mathcal{G}$ adapted dividend processes $\mathbf{D}_{\mathbf{x}}$ (the assets $A_{j}$ are $\mathcal{F}$ adapted). Our aim is to find the optimized, $\mathcal{G}$ adapted, positive consumption stream $\mathbf{c}(\mathbf{w}, \mathbf{x})=$ $\mathbf{c}(0,0, \mathbf{w}, \mathbf{x})=\mathbf{w}+\mathbf{D}_{\mathbf{x}}=\left(c_{t}\right)_{t=0, \ldots . T}$ in this enlarged $\sigma$-algebra (or equivalently in the incomplete market $(\mathscr{M}, \mathcal{G})$ ). In analogy to Theorem 3.1 we obtain the following theorem in the incomplete market situation. The following simple lemma is an immediate consequence of the definition of upper hedging price.

LEMMA 3.6. There exists a G-adapted portfolio strategy $\mathbf{x}$ such that the consumption stream $\mathbf{c}(\mathbf{w}, \mathbf{x})$ is positive if and only if

$$
w_{0}>(-\mathbf{w})^{u} \text {. }
$$

Note that the optimal consumption streams for endowment processes $\mathbf{w}$ and $\mathbf{c}(\mathbf{w}, \mathbf{x})$ are identical because $\mathbf{c}(\mathbf{w}, \mathbf{x}+\mathbf{y})=\mathbf{c}\left(\mathbf{w}+\mathbf{D}_{\mathbf{x}}, \mathbf{y}\right)$. Thus, if (3.12) is fulfilled, we may assume that the endowment process is positive. Note that if the optimal consumption stream, maximizing the utility, is not strictly positive then we have a boundary (not interior) solution, and the optimal consumption stream will not satisfy the first order conditions. It is well known that the utility maximization problem

$$
\max E\left[\sum_{t=0}^{T} u\left(c_{t}\right)\right]
$$

with a positive endowment stream does have a unique, positive optimal solution if the utility function $u$ satisfies the so-called Inada condition at zero:

$$
\lim _{c \rightarrow 0} u^{\prime}(c)=\infty,
$$

see Karatzas-Zitkovic [21] for a general result and Schachermeyer [31], Theorem 2.18, for the simple, finite dimensional case that we analyze here. Obviously, our utility function $u=(1-\gamma)^{-1} c^{1-\gamma}$ satisfies the Inada condition and thus the uniqueness and existence of the optimal consumption stream immediately follow.

Theorem 3.7. Suppose that (3.12) holds. Then there exists a unique, G adapted, positive, optimal consumption stream

$$
c_{t}=w_{t}+D_{\mathbf{x}, t}=w_{t}+X_{t}-E\left[\frac{X_{t+1} M_{t+1}}{M_{t}} \mid \mathcal{G}_{t}\right]>0
$$

for the financial market $(\mathcal{M}, \mathcal{G})$ that maximizes $(2.10)$. It is determined through the first order conditions

$$
e^{-\rho} E\left[c_{t}^{-\gamma} M_{t}^{-1} \mid \mathcal{H}_{t}\right]=c_{t-1}^{-\gamma} M_{t-1}^{-1} .
$$


That is, the process $\left(e^{-\rho t} c_{t}^{-\gamma} M_{t}^{-1}, t=0, \ldots, T\right)$ is a martingale with respect to the shifted filtration $\left(\mathcal{H}_{t+1}\right)_{t}$.

The key difference between Theorem 3.7 and general existence results (such as in Karatzas-Zitkovic [21]) is that the special market structure, encoded in the filtration $\mathcal{H}$ and the aggregate state price densities $\mathbf{M}$, generates a remarkable form (3.16) of first order conditions, that will allow us to solve them explicitly by a recusive procedure.

Recall that, by Theorem 3.1, the marginal utility $e^{-\rho t} c_{t}^{-\gamma}$, coincides with the aggregate state price density $M_{t}$. When markets are incomplete, the agent is not able to achieve this identity because the optimal consumption stream is not $\mathcal{F}$-adapted, and $\mathbf{M}$ is $\mathcal{F}$-adapted. But the agent is able to perfectly replicate any event in the hedgeable filtration $\mathcal{H}$. Thus, he wants to make the marginal utility as close as possible to $M_{t}$, given the state in $\mathcal{H}_{t}$. The agent achieves this by making the quotient $e^{-\rho t} c_{t}^{-\gamma} M_{t}^{-1}$ a martingale.

As a first application of the first order conditions of Theorem 3.7, we prove the following limit result:

THEOREM 3.8. We have

$$
\lim _{\gamma \rightarrow \infty} \pi_{0}=\mathbf{Y}^{u}
$$

\section{INDUCTIVE STRUCTURE FOR THE WEALTH PROCESS}

Note that for the complete market case the optimal $\mathcal{F}$ adapted consumption stream for the $\mathcal{F}$ adapted endowment process $\mathbf{w}$ is given explicitly in (3.2).

The incomplete market situation $(\mathcal{M}, \mathcal{G})$ is more involved. Here, we assume, on the one hand, that the $\mathcal{F}$ adapted endowment process $\mathbf{w}$ becomes $\mathcal{G}$ adapted through transformation (2.15). On the other hand, we choose our portfolio strategy $\mathbf{x}$ according to the information $\mathcal{G}$. Hence, we consider optimal consumption streams in an incomplete market $(\mathcal{M}, \mathcal{G})$ (though the original endowment process w and the assets remain unchanged $\mathcal{F}$ adapted processes). For this incomplete market situation we give an inductive structure which allows explicitly for calculating the optimal consumption stream within $(\mathcal{M}, \mathcal{G})$. This is then applied to the static premium $\Pi_{0}$ case.

We therefore need several defintions. Choose $x \in \mathbb{R}$. Define $G_{T+1}(x, \mathbf{w}) \equiv 0$. For $t=T, \ldots, 1$, we define inductively the $\mathcal{H}_{t}$-measurable random function $F_{t}(x, \mathbf{w})$ by the solution of

$$
e^{-\rho} E\left[\left(w_{t}+F_{t}(x, \mathbf{w})-G_{t+1}\left(F_{t}(x, \mathbf{w}), \mathbf{w}\right)\right)^{-\gamma} M_{t}^{-1} \mid \mathcal{H}_{t}\right]=\left(w_{t-1}+x\right)^{-\gamma} M_{t-1}^{-1},
$$

the $G_{t-1}$-measurable random function $G_{t}(x, \mathbf{w})$ by

$$
G_{t}(x, \mathbf{w})-E\left[\frac{M_{t}}{M_{t-1}} F_{t}\left(x-G_{t}(x, \mathbf{w}), \mathbf{w}\right) \mid G_{t-1}\right]=0,
$$


and the $\mathcal{H}_{t}$-measurable random function $H_{t}$ is defined by

$$
H_{t}(x, \mathbf{w})=F_{t}\left(x-G_{t}(x, \mathbf{w}), \mathbf{w}\right) .
$$

Lemma 4.1. Assume $w_{t}>0$, a.s., for all $t=0, \ldots, T$. Then the random functions defined in (4.1)-(4.3) exist.

\section{REMARKS 4.2}

- The assumptions of Lemma 4.1 can be relaxed (see Lemma C.1 below). These weaker assumptions give a first lower bound $\left(w+\pi_{0}\right)_{0}^{i n f}>0$ on the static premium $\Pi_{0}$ which can be understood as a regulatory solvency condition.

- The functions $F_{t}$ and $G_{t}$ are jointly convex in the variables $(x, \mathbf{w})$. See Malamud-Trubowitz [22] for a proof. This is not needed in the sequel.

Now we are ready to formulate our key result:

THEOREM 4.3. (CONSTRUCTION OF THE OPTIMAL CONSUMPTION STREAM)

The optimal financial wealth process $\left(X_{t}\right)_{t=0, \ldots, T}$ for the $G$ adapted endowment process $\mathbf{w}$ is given iteratively by $X_{0}=0$ and

$$
X_{t}=H_{t}\left(X_{t-1}, \mathbf{w}\right) \text { for } t=1, \ldots, T .
$$

Having introduced the two sets of functions $\left(F_{t}\right)_{t}$ and $\left(G_{t}\right)_{t}$ the maximization problem for finding optimal consumption streams decouples in a "miraculous" way which allows for the iterative calculation of the wealth process $X_{t}$ (Theorem 4.3) and through Theorem 3.7 of optimal consumption streams. Hence, Theorem 4.3 allows for the calculation of the static premium $\Pi_{0}$. We require that the expected optimal utility remains unchanged when adding the insurance claims $\mathbf{Y}$. This means that we evaluate the optimal consumption stream for the complete market $\left(\mathrm{cm}_{t}\right)_{t}$ (given by (2.13)) and the optimal consumption stream generated by the wealth process

$$
X_{t}=H_{t}\left(X_{t-1}, \mathbf{w}+\Pi_{0}-\mathbf{Y}\right),
$$

and determine $\pi_{0}$ such that their expected utility is equal. For an example and the computational implementation we refer to Section 6 .

\section{BORROWING CONSTRAINTS}

In Proposition 2.13 we have seen that the sum of the discounted $\mathcal{G}_{0}$-measurable annual premia is equal to the static insurance premium $\pi_{0}$. This makes perfect sense from a mathematical point of view, but it is not reasonable from a practical point of view. It basically means that we can borrow future premium from 
third party without any restrictions. Of course, in practice there are restrictions for borrowing money from third party, e.g. imposed by the regulator. That is, we introduce borrowing constraints, which allow borrowing money only up to a certain threshold $a$ and therefore an insurance contract with annual premia becomes more expensive than a contract with one single premium installment. This exactly reflects that the regulator rather likes to see the real cash amount on the balance sheet of the insurance company than a receivable for future, fixed premia income.

The borrowing constraints are introduced as follows. Choose $a \geq 0$. Then

$$
B_{a}=\left\{\mathbf{x} \text { trading strategy; } E\left[\frac{M_{t+1} X_{t+1}}{M_{t}} \mid G_{t}\right] \geq-a \text { for all } t=0, \ldots, T-1\right\} .
$$

Note that this exactly reflects that we restrict our trading strategies $\mathbf{x}$ such that we do not borrow too much from third party.

Moreover, we define for the premia flow $\Pi=\left(\Pi_{0}, \ldots, \Pi_{T}\right)$

$$
U^{\max , a}(\mathbf{w}+\Pi-\mathbf{Y})=\max _{\mathbf{x} \in B_{a}} U\left(\mathbf{w}+\Pi+D_{\mathbf{x}}-\mathbf{Y}\right) .
$$

DEFINITION 5.1. Let $\Pi_{0}^{a}=\left(\pi_{0}^{a}, 0, \ldots, 0\right)$ be the static premium under the borrowing constraints $B_{a}$, that is, the unique $\mathcal{G}_{0}$-measurable solution to

$$
U^{\max , a}\left(\mathbf{w}+\Pi_{0}^{a}-\mathbf{Y}\right)=U^{\max }(\mathbf{w}) .
$$

Similarly, we define the constant premia flow $\Pi_{a n n}^{a}=\left(0, \pi_{a n n}^{a}, \ldots, \pi_{\text {ann }}^{0}\right)$ under the borrowing constraints $B_{a}$ to be the unique $\mathcal{G}_{0}$-measurable solution to

$$
U^{\max , a}\left(\mathbf{w}+\Pi_{a n n}^{a}-\mathbf{Y}\right)=U^{\max }(\mathbf{w}) .
$$

Note that without borrowing constraints $a=+\infty$ and we use the convention $\pi_{0}^{\infty}=\pi_{0}$ and $\pi_{a n n}^{\infty}=\pi_{a n n}$.

Note that, in complete analogy with Theorem 2.11, we should first understand whether the just defined premia exist. We will need some definitions.

Definition 5.2. Let $\mathbf{Y}$ be a claims payment stream. Set $Y_{T+1}^{\text {sup }, a} \equiv 0$. We define inductively an $\mathcal{H}$ adapted process, $t=1, \ldots, T+1$, by

$$
Y_{t-1}^{\text {sup }, a}=\operatorname{esssup}\left[Y_{t-1}+\max \left\{E\left[\frac{M_{t}}{M_{t-1}} Y_{t}^{\text {sup }, a} \mid \mathcal{G}_{t-1}\right],-a\right\} \mid \mathcal{H}_{t-1}\right] .
$$

Note that, by construction, $Y_{0}^{\text {sup, } a}$ is monotone decreasing with $a$.

Recall Definition 2.7. An immediate consequence is: 
LEMMA 5.3. $Y^{u, a}=Y_{0}^{\text {sup, } a}$ is the upper hedging price under the borrowing constraints $a$. Consequently, the budget set $B_{a}$ is nonempty if and only if

$$
w_{0}>(-\mathbf{w})^{u, a} \text {. }
$$

The following direct analog of Theorem 2.11 is true:

THEOREM 5.4. Let $\mathbf{w}$ be the endowment stream of the company and $\mathbf{Y}$ the claims stream. The following holds true:

The market consistent static premium $\Pi_{0}^{a}$ exists if and only if $\gamma<1$ and

$$
\lim _{\pi_{0} \rightarrow(\mathbf{Y}-\mathbf{w})^{u, a}-w_{0}} U^{\max , a}\left(\mathbf{w}+\Pi_{0}-\mathbf{Y}\right)<U^{\max }(\mathbf{w})
$$

or $\gamma>1$.

Similarly, let

$$
\pi_{\text {ann }}^{\min }=\left\{\min \pi: \exists \mathbf{x} \in B_{a}, \pi-y_{t}+w_{t}+D_{\mathbf{x}, t} \geq 0 \text { for all } t\right\}
$$

be the minimal annual premium, sufficient to upper hedge the claims stream. Then, the market consistent annual premium $\Pi_{\text {ann }}^{a}$ exists if and only if $\gamma<1$ and

$$
\lim _{\pi_{a n n} \rightarrow \pi_{a n n}^{\min }} U^{\max , a}\left(\mathbf{w}+\Pi_{a n n}-\mathbf{Y}\right)<U^{\max }(\mathbf{w})
$$

or $\gamma>1$.

The proof of this result is completely analogous to that of Theorem 2.11 .

PROPOSITION 5.5. The premia $\pi_{0}^{a}$ and $\pi_{\text {ann }}^{a}$ are monotone decreasing and convex functions of a. Furthermore

$$
\pi_{\text {ann }}^{a} \sum_{t=1}^{T} E\left[M_{t}\right] \geq \pi_{0}^{a} \geq \pi_{0}=\pi_{\text {ann }} \sum_{t=1}^{T} E\left[M_{t}\right]
$$

for all $a \in[0, \infty)$.

THEOREM 5.6. Let (5.6) be fulfilled. Under the borrowing constraint (5.1) there exists a unique, nonnegative $\mathcal{G}_{t}$-adapted process $\left(\lambda_{t}\right)_{t}$ such that

$$
e^{-\rho} E\left[c_{t}^{-\gamma} M_{t}^{-1} \mid \mathcal{H}_{t}\right]=c_{t-1}^{-\gamma} M_{t-1}^{-1}-\lambda_{t-1} M_{t-1}^{-1},
$$

and

$$
\lambda_{t}\left(M_{t}^{-1} E\left[M_{t+1} X_{t+1} \mid \mathcal{G}_{t}\right]+a\right)=0 \text { for all } t .
$$


In particular, the marginal utility process $e^{-\rho t} c_{t}^{-\gamma} M_{t}^{-1}$ relative to the aggregate state price densities is a super-martingale.

We now describe a recursive construction, analogous to that in the case without constraints. We define $\tilde{G}_{T+1} \equiv 0$. Then for $t \leq T$ and $\lambda \geq 0$ let $\tilde{F}_{t}(x, \lambda, \mathbf{w})$ be a $\mathcal{H}_{t}$ adapted solution to

$$
\begin{aligned}
e^{-\rho} E[ & {\left[\left(w_{t}+\tilde{F}_{t}(x, \lambda, \mathbf{w})-\tilde{G}_{t+1}\left(\tilde{F}_{t}(x, \lambda, \mathbf{w}), \mathbf{w}\right)\right)^{-\gamma} M_{t}^{-1} \mid \mathcal{H}_{t}\right] } \\
& =\left[\left(w_{t-1}+x\right)^{-\gamma}-\lambda\right] M_{t-1}^{-1} .
\end{aligned}
$$

Moreover, we define the $\mathcal{G}_{t-1}$-measurable random function $\tilde{G}^{(1)}(x, \mathbf{w})$ to be a solution to

$$
\tilde{G}_{t}^{(1)}(x, \mathbf{w})-E\left[\frac{M_{t}}{M_{t-1}} \tilde{F}_{t}\left(x-\tilde{G}_{t}^{(1)}(x, \mathbf{w}), 0, \mathbf{w}\right) \mid \mathcal{G}_{t-1}\right]=0 .
$$

The $\mathcal{G}_{t-1}$-measurable random function $\Lambda_{t}(x)$ is a solution to

$$
E\left[\frac{M_{t}}{M_{t-1}} \tilde{F}_{t}\left(x+a, \Lambda_{t}(x), \mathbf{w}\right) \mid \mathcal{G}_{t-1}\right]=-a
$$

if $\tilde{G}_{t}^{(1)}(x, \mathbf{w})<-a$, and $\Lambda_{t}(x)=0$ if $\tilde{G}_{t}^{(1)}(x, \mathbf{w}) \geq-a$.

Then, we define the $\mathcal{G}_{t-1}$-measurable random function

$$
\tilde{G}_{t}(x, \mathbf{w})= \begin{cases}\tilde{G}_{t}^{(1)}(x, \mathbf{w}) & \text { if } \tilde{G}_{t}^{(1)}(x, \mathbf{w}) \geq-a, \\ -a & \text { otherwise }\end{cases}
$$

Finally, we define

$$
\tilde{H}_{t}(x, \mathbf{w})=\tilde{F}_{t}\left(x-\tilde{G}_{t}(x, \mathbf{w}), \Lambda_{t}(x), \mathbf{w}\right) .
$$

In analogy to Lemmas 4.1 and C.1 we prove the following lemma under the borrowing constraints.

LEMMA 5.7. The random function $\tilde{F}_{t}$ and $\tilde{G}_{t}$ exist if and only if (5.6) is fulfilled.

Hence we obtain the following construction for the optimal consumption stream under budget constraint.

THEOREM 5.8. There exists a unique, optimal consumption stream under the budget constraints. The optimal financial wealth process $\left(X_{t}\right)_{t=0, \ldots, T}$ under the budget constraint $B_{a}$ is given iteratively by $X_{0}=0$ and

$$
X_{t}=\tilde{H}_{t}\left(X_{t-1}, \mathbf{w}\right) \text { for } t=1, \ldots, T .
$$


Note that, superficially, there might be problems with the uniqueness of the recursively constructed functions $\tilde{G}_{t}$ and $\tilde{F}_{t}$ because, by contrast with their counterparts in Theorem 4.3, they are not strictly monotone. It is possible to directly show that the functions are unique. However, we do not need it since the uniqueness follows from the Kuhn-Tucker Theorem (see, Ingersoll [20]). In fact, the Kuhn-Tucker Theorem tells that if the utility function is strictly concave, then there exists a unique optimal consumption stream satisfying the first order conditions (5.11). Thus, if the functions $\tilde{G}_{t}$ or $\tilde{F}_{t}$ were non-unique, Theorem 5.8 would imply non-uniqueness of the optimal stream, contradicting the Kuhn-Tucker Theorem.

Theorem 5.8 is the analogy to Theorem 4.3 under the borrowing constraints. That is, in analogy to before we can iteratively construct the optimal consumption stream using the additional set of functions $\tilde{F}_{t}$ and $\tilde{G}_{t}$, and hence can calculate the premium under borrowing constraints.

\section{EXAMPLE: STATIC PREMIUM}

In this section we give a toy example to describe how the inductive structure is implemented computationally. Therefore we need several steps:

(1) choose a model for the state price densities that generates reasonable asset prices that can be calibrated;

(2) choose a basis of assets which span the whole financial market subspace. The hedging is then obtained by trading only these basis assets;

(3) define a model for the insurance claims;

(4) find a numerical algorithm for calculating market consistent premia.

\subsection{Financial market}

For (1), we choose the simplest discrete time Markov model for state price densities that is rich enough to generate a reasonable behaviour (for a discussion see Filipovic-Zabczyk [12]). Assume that there exists an underlying finite state Markov process $S_{t}$ taking values $s_{1}, \ldots, s_{n}$ with transition probabilities $p_{i, j}$, $i, j=1, \ldots, n$, and initial probabilities $p_{0, j}, j=1, \ldots, n$. Then, we define the multiplicative process (state price density) by

$$
M_{t}=S_{1} \cdots S_{t} .
$$

The price $B(t, \tau)$ at time $t$ of a riskfree bond maturing at time $\tau$ is

$$
B(t, \tau)=M_{t}^{-1} E\left[M_{\tau} \mid \mathcal{G}_{t}\right]=E\left[S_{t+1} \cdots S_{\tau} \mid S_{t}\right] .
$$

As a model for a risky asset we choose an asset whose dividend process $D_{t}$ is of the following form: for a positive function $f$,

$$
\left(D_{t}\right)_{t \geq 1}=f\left(S_{1}\right) \cdots f\left(S_{t}\right) \text {. }
$$


As a concrete example we consider $T=2$ periods and let $n=3$. The spanning number of the generated filtration $\mathcal{F}$ is also $n=3$ and three assets suffice to make the market complete. We choose these assets to be the one and two period riskfree zero coupon bonds and a risky asset. Let $q_{2}\left(s_{i}\right)$ be the price of the risky asset at time 2 if we are in state $s_{i}$. We do not specify the further development of the dividend and price process of the risky asset after $t=2$. Then, by no arbitrage, we have the following prices at time $t=1$ in state $s_{i}$

$$
q_{1}\left(s_{i}\right)=\sum_{j=1}^{n} p_{i, j}\left(q_{2}\left(s_{j}\right)+D_{2}\left(s_{j}\right)\right) s_{j}
$$

and at time $t=0$

$$
q_{0}=\sum_{j=1}^{n} p_{0, j}\left(q_{1}\left(s_{j}\right)+D_{1}\left(s_{j}\right)\right) s_{j}
$$

For the zero coupon bonds we have

$$
B(0,1)=\sum_{j=1}^{n} p_{0, j} s_{j}, \text { and } B(0,2)=\sum_{j=1}^{n} p_{0, j} s_{j}\left(\sum_{k=1}^{n} p_{j} s_{k}\right) .
$$

Similarly, at time $t=1$,

$$
B(1,2)\left(s_{i}\right)=\sum_{j=1}^{n} p_{i, j} s_{j}
$$

\subsection{Insurance claims}

The insurance claims $Y_{1}, Y_{2}$ generating the filtration $\mathcal{G}$ are chosen as follows. We imagine that, conditional on state $s_{3}$, there are two possible states $s_{3,1}$ and $s_{3,2}$ for the insurance claim. Thus, the branching number of the event tree corresponding to $\mathcal{G}$ is four. Consequently, the market is incomplete and we can only hedge a part of the insurance claims. We assume that the states $s_{3,1}$ and $s_{3,2}$ have the same probability and, since we need Assumption 3 to be satisfied, we also need that the transition probabilities $p_{i, j}$ are independent of the idiosyncratic state $s_{3,1}$ and $s_{3,2}$.

\subsection{Implementation}

The inductive structure gives the financial wealths $X_{1}$ and $X_{2}$ at time $t=1,2$. The main difficulty in the implementation is that the functions $F_{2}, G_{2}, F_{1}$ and $G_{1}$ are only defined implicitly and each function in the series depends on the previous ones. We choose the following simple algorithm: we compute the values of the function $F_{2}$ on a grid with a small step $\varepsilon$. Then, we proceed 
similarly for $G_{2}$, using linear interpolation for $F_{2}$ between the grid points. We repeat this procedure inductively and construct the functions $F_{1}$ and $G_{1}$.

\subsection{Numerical calculation}

We assume that the insurance company is endowed initially with $w_{0}=2$ and $w_{1}=w_{2}=0$.

We choose the following parameters $\left(s_{1}, s_{2}, s_{3}\right)=(0.99,1.01,1.04)$. The transition probabilities are given by $p_{0, j}=1 / 3, j=1,2,3$, and for $i \geq 1$

$$
\left(p_{i, j}\right)_{i, j=1,2,3}=\left(\begin{array}{lll}
1 / 3 & 1 / 3 & 1 / 3 \\
1 / 5 & 2 / 5 & 2 / 5 \\
1 / 5 & 2 / 5 & 2 / 5
\end{array}\right) .
$$

Then, the zero coupon bond prices at time 0 are $B(0,1)=1.0133$ and $B(0,2)=1.03$. The bond prices at time 1 are given by $B(1,2)\left(s_{1}\right)=1.0133$ and $B(1,2)\left(s_{2}\right)=B(1,2)\left(s_{3}\right)=1.018$.

We assume that $Y_{1}$ and $Y_{2}$ are independent and identically distributed, taking values

$$
y\left(s_{1}\right)=0.25, y\left(s_{2}\right)=0.3
$$

in the states $s_{1}$ and $s_{2}$ and taking values 0.5 and 2 with equal probabilities, conditioned on the state $s_{3}$.

Using Theorem 3.8 we find

$$
1.292 \leq \pi_{0} \leq 1.852 .
$$

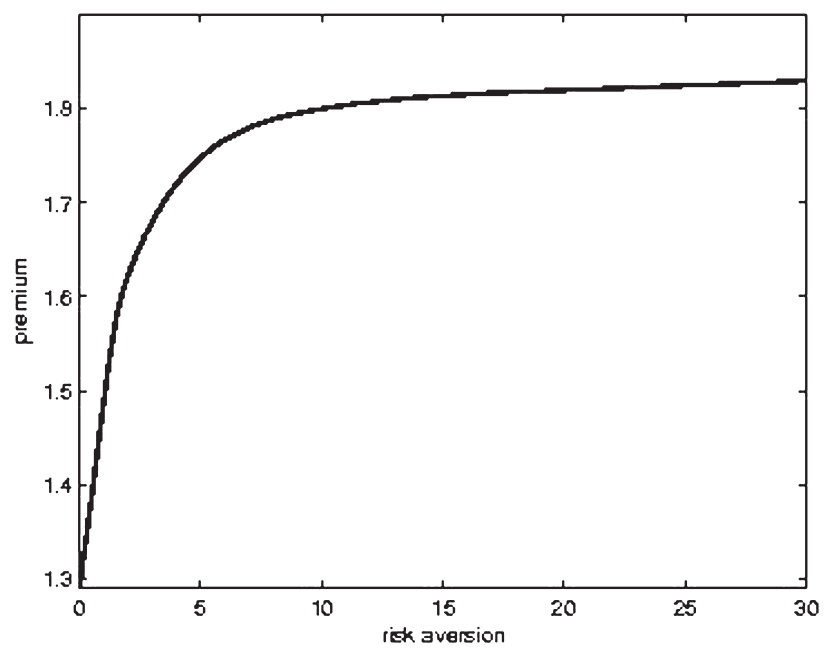


The difference $\pi_{0}-1.292$ corresponds to the risk premium depending on the risk aversion $\gamma$ chosen.

The picture suggests that the premium $\pi_{0}$ is an increasing function in $\gamma$. However, further numerical calculations in other examples indicate that this is not true, in general.

\section{A. Proofs of Section 2}

Proof of Lemma 2.6. Observe the $u(c)=c^{1-\gamma} /(1-\gamma)$ is a concave function. Henceforth, for any two portfolio strategies $\mathbf{x}_{1}$ and $\mathbf{x}_{2}$ and any two surplus processes $\mathbf{w}_{1}$ and $\mathbf{w}_{2}$ we have

$$
\begin{aligned}
& U^{\max }\left(\frac{\mathbf{w}_{1}+\mathbf{w}_{2}}{2}\right) \geq U\left(\frac{\mathbf{w}_{1}+\mathbf{w}_{2}}{2}+D_{\left(\mathbf{x}_{1}+\mathbf{x}_{2} / 2\right)}\right) \\
& =U\left(\frac{\left(\mathbf{w}_{1}+\mathbf{D}_{\mathbf{x}_{1}}\right)+\left(\mathbf{w}_{1}+\mathbf{D}_{\mathbf{x}_{2}}\right)}{2}\right) \\
& \geq \frac{1}{2}\left(U\left(\mathbf{w}_{1}+\mathbf{D}_{\mathbf{x}_{1}}\right)+U\left(\mathbf{w}_{2}+\mathbf{D}_{\mathbf{x}_{2}}\right)\right) \text {. }
\end{aligned}
$$

Taking the supremum over $\mathbf{x}_{1}, \mathbf{x}_{2}$ we get the concavity (the increasing property follows by the same lines).

Suppose that $U$ is strictly monotone increasing and the maximum is always achieved, and let $\mathbf{w}_{1} \leq \mathbf{w}_{2}$ a.s., and $\mathbf{w}_{1}<\mathbf{w}_{2}$ with positive probability. Then

$$
U^{\max }\left(\mathbf{w}_{1}\right)=U\left(\mathbf{w}_{1}+\mathbf{D}_{\mathbf{x}}\right)<U\left(\mathbf{w}_{2}+\mathbf{D}_{\mathbf{x}}\right) \leq U^{\max }\left(\mathbf{w}_{2}\right) .
$$

Thus, $U^{\max }$ is also strictly increasing.

If the market is complete, Lemma 2.3 implies that any consumption stream satisfying

$$
E\left[\sum_{t=0}^{T} c_{t}\right]=W
$$

is achievable by trading. The required assertion follows.

Proof of Lemma 2.9. By Lemma 2.3, we may assume that the company's endowment stream is given by $(W, 0, \ldots, 0)$. By definition, $(2.23)$ is necessary and sufficient for the existence of a portfolio strategy $\mathbf{x}$ such that

$$
c_{t}=D_{\mathbf{x}, t}-Y_{t}>0
$$

for all $t \geq 1$ and 


$$
c_{0}=\pi_{0}+W-D_{\mathbf{x}, 0}>0 .
$$

Thus, (2.23) is necessary and sufficient for the maximal utility to be defined. Recall that $c_{0}=\pi_{0}+w_{0}-D_{\mathbf{x}, 0}$. Furthermore, by the definition of the upper hedging price, any strategy $\mathbf{x}$ satisfying (A.3) fulfills $D_{\mathbf{x}, 0}>\mathbf{Y}^{u}$. Thus, $0<c_{0}<$ $\pi_{0}-\left(\mathbf{Y}^{u}-W\right)$ and, therefore,

$$
\lim _{\pi_{0} \rightarrow\left(\mathbf{Y}^{u}-W\right)} c_{0}=0 .
$$

Let $\gamma>1$. Then

$$
(1-\gamma) U^{\max }>c_{0}^{1-\gamma} \rightarrow \infty
$$

and therefore $(1-\gamma) U^{\max } \rightarrow \infty$.

Let now $\pi_{0} \rightarrow \infty$. Then, as $\pi_{0}$ becomes large, any portfolio strategy becomes feasible for the company. Let $\mathbf{x}$ be any upper hedging strategy for $\mathbf{Y}-\mathbf{w}$. Fix a large $N>0$. Since the market $(\mathcal{M}, \mathcal{F})$ is complete, there exists a portfolio strategy $\mathbf{z}^{N}$ such that $D_{\mathbf{z}^{N, t}}=N$ for all $t \geq 1$. If $\pi_{0}$ is so large that $\pi_{0}+D_{\mathbf{z}^{N+} \mathbf{x}, 0}>N$ then

$$
U^{\max }\left(\Pi_{0}+\mathbf{w}-\mathbf{Y}\right) \geq U\left(\Pi_{0}+\mathbf{w}+D_{\mathbf{z}^{N}+\mathbf{x}}-\mathbf{Y}\right)>(1-\gamma)^{-1}(T+1) N^{1-\gamma} e^{-\rho T}
$$

and, consequently,

$$
0<(1-\gamma) U^{\max }\left(\Pi_{0}+\mathbf{w},-\mathbf{Y}\right) \leq(T+1) N^{1-\gamma} e^{-\rho T} \rightarrow 0
$$

as $N \rightarrow \infty$. The same argument implies for $\gamma<1$ that

$$
\lim _{\pi_{0} \rightarrow \infty}(1-\gamma) U^{\max }\left(\Pi_{0}+\mathbf{w}-\mathbf{Y}\right)=\infty
$$

as $N \rightarrow \infty$.

Let us now consider the limit $\pi_{0} \downarrow \mathbf{Y}^{u}-W$ when $\gamma<1$. Note that the limit

$$
\lim _{\pi_{0} \downarrow \mathbf{Y}^{u}-W} U^{\max }\left(\Pi_{0}+\mathbf{w}-\mathbf{Y}\right)
$$

exists because $U^{\max }$ is monotone increasing. As we have shown above, in this case $c_{0} \rightarrow 0$ but, since $\gamma<1, c_{0}^{1-\gamma} \rightarrow \infty$, too. Note that any achievable consumption stream $\mathbf{c}=\mathbf{w}+\mathbf{D}_{\mathbf{x}}$ satisfies

$$
E\left[\sum_{t=0}^{T} c_{t} M_{t}\right]=W+\pi_{0} .
$$

Thus, since the probability space is finite and $\mathbf{M}$ is strictly positive, there exists a constant $K$ such that $0<c_{t}<K\left(W+\pi_{0}\right)$ for all $t$. Consequently, any dividend process $D_{\mathbf{x}, t}$ satisfies 


$$
\min Y_{t}<D_{\mathbf{x}, t}<K\left(W+\pi_{0}\right)+\max Y_{t} .
$$

Let $\mathbf{D}_{\mathbf{x}}(\pi)$ be the optimal portfolio strategy, corresponding to the premium $\pi$. Consequently, the set of the dividend processes is bounded. Let us send $\pi_{0} \rightarrow$ $\mathbf{Y}^{u}-W$. Then, as we have shown above, $c_{0} \rightarrow 0$ and, consequently $D_{\mathbf{x}, 0}\left(\pi_{0}\right) \rightarrow \mathbf{Y}^{u}$. Since, by the above, the set of dividend processes is pre-compact, we can, passing to subsequences, assume that $\mathbf{D}_{\mathbf{x}}\left(\pi_{0}\right) \rightarrow \mathbf{D}_{\mathbf{x}}\left(\mathbf{Y}^{u}\right)$. Consequently,

$$
\lim _{\pi_{0} \downarrow \mathbf{Y}^{u}-W} U^{\max }\left(\Pi_{0}+\mathbf{w}-\mathbf{Y}\right)=U^{\max }\left(\Pi_{0}+\mathbf{w}+D_{\mathbf{x}}\left(\mathbf{Y}^{u}\right)-\mathbf{Y}\right)>0 .
$$

The last inequality holds because $\Pi_{0}+D_{\mathbf{x}}\left(\mathbf{Y}^{u}\right)-\mathbf{Y}$, can not be identically zero when $\mathbf{Y}$, is not replicable.

Proof of Theorem 2.11. Due to the monotonicity of $U$ we have

$$
U^{\max }(\mathbf{w}-\mathbf{Y}) \leq U^{\max }(\mathbf{w}) .
$$

By definition we want to achieve (2.26). The left-hand side of (2.26) is monotone increasing in $\pi_{0}$ and converges to infinity for $\pi_{0} \rightarrow \infty$ for $\gamma>1$, hence there exists a $\mathcal{G}_{0}$-measurable solution $\pi_{0}$ that satisfies (2.26). Using that $U^{\max }$ is strictly increasing we obtain the uniqueness of $\pi_{0}$ (the same argument holds true for $\gamma<1$ as long as (2.27) is satisfied).

Moreover, for the two claims $\mathbf{Y}^{(1)}$ and $\mathbf{Y}^{(2)}$ we have

$$
\begin{aligned}
& U^{\max }\left(\mathbf{w}+\frac{\Pi_{0}\left(\mathbf{Y}^{(1)}\right)+\Pi_{0}\left(\mathbf{Y}^{(2)}\right)}{2}-\frac{\mathbf{Y}^{(1)}+\mathbf{Y}^{(2)}}{2}\right) \\
& \quad \geq \frac{1}{2}\left[U^{\max }\left(\mathbf{w}+\Pi_{0}\left(\mathbf{Y}^{(1)}\right)-\mathbf{Y}^{(1)}\right)+U^{\max }\left(\mathbf{w}+\Pi_{0}\left(\mathbf{Y}^{(2)}\right)-\mathbf{Y}^{(2)}\right)\right] \\
& \quad=U^{\max }(\mathbf{w})=U^{\max }\left(\mathbf{w}+\Pi_{0}\left(\left(\mathbf{Y}^{(1)}+\mathbf{Y}^{(2)}\right) / 2\right)-\frac{\mathbf{Y}^{(1)}+\mathbf{Y}^{(2)}}{2}\right)
\end{aligned}
$$

and, by monotonicity,

$$
\frac{\pi_{0}\left(\mathbf{Y}^{(1)}\right)+\pi_{0}\left(\mathbf{Y}^{(2)}\right)}{2} \geq \pi_{0}\left(\left(\mathbf{Y}^{(1)}+\mathbf{Y}^{(2)}\right) / 2\right) .
$$

Similarly, if $\mathbf{Y}^{(1)} \leq \mathbf{Y}^{(2)}$ then, by definition,

$$
U^{\max }\left(\mathbf{w}+\Pi_{0}\left(\mathbf{Y}^{(1)}\right)-\mathbf{Y}^{(1)}\right) \geq U^{\max }\left(\mathbf{w}+\Pi_{0}\left(\mathbf{Y}^{(1)}\right)-\mathbf{Y}^{(2)}\right),
$$

that is $\pi_{0}\left(\mathbf{Y}^{(1)}\right) \leq \pi_{0}\left(\mathbf{Y}^{(2)}\right)$.

The inequality $\pi_{0}>\mathbf{Y}^{u}-W$ should always hold because without it the value function is not defined, see (2.23). Suppose now that $\pi_{0}>\mathbf{Y}^{u}$. Then, there exists 
a portfolio strategy $\mathbf{x}$ such that $D_{\mathbf{x}, t}>\mathbf{Y}$ and $\pi_{0}+D_{\mathbf{x}, 0}>0$. Consequently, for any other portfolio strategy $\mathbf{z}$,

$$
U\left(\Pi_{0}+\mathbf{w}-\mathbf{Y}+\mathbf{D}_{\mathbf{x}+\mathbf{z}}\right)=U\left(\Pi_{0}+\mathbf{w}-\mathbf{Y}+\mathbf{D}_{\mathbf{x}}+\mathbf{D}_{\mathbf{z}}\right)>U\left(\mathbf{w}+\mathbf{D}_{\mathbf{z}}\right)
$$

and, consequently, $U^{\max }\left(\Pi_{0}+\mathbf{w}-\mathbf{Y}\right)>U^{\max }(\mathbf{w})$. This gives a contradiction.

Proof of Proposition 2.13. The proof is completely analogous to the derivations in (D.7)-(D.9).

Lemma A.1 (Hölder's INequality) Let $\gamma>0$. We have

$$
\frac{1}{1-\gamma} E\left[X^{\gamma} Y^{1-\gamma}\right] \leq \frac{1}{1-\gamma} E[X]^{\gamma} E[Y]^{1-\gamma} .
$$

In particular, for any positive vectors $\left(a_{i}\right)_{i},\left(b_{i}\right)_{i}$

$$
\frac{1}{1-\gamma} \sum_{i} a_{i}^{1-\gamma} b_{i}^{\gamma} \leq \frac{1}{1-\gamma}\left(\sum_{i} a_{i}\right)^{1-\gamma}\left(\sum_{i} b_{i}\right)^{\gamma} .
$$

Proof. For $\gamma<1$ this is just Hölder's inequality. If $\gamma>1$, define $Z=X^{1-\gamma} Y^{\gamma}$. Then $Y=X^{1-\gamma^{-1}} Z^{\gamma^{-1}}$ and Hölder's inequality implies

$$
E[Y]=E\left[X^{1-\gamma^{-1}} Z^{\gamma^{-1}}\right] \leq E[X]^{1-\gamma^{-1}} E\left[X^{1-\gamma} Y^{\gamma}\right]^{\gamma^{-1}} .
$$

Potentiating this inequality into power $\gamma$, completes the proof.

Proof of Lemma 2.14. In the complete market $(\mathcal{M}, \mathcal{F})$ situation the optimal consumption stream is given by (see Theorem 3.1)

$$
\mathrm{cm}_{t}=c_{0} e^{-\rho t / \gamma} M_{t}^{-1 / \gamma} \text {. }
$$

This implies for $\mathcal{F}$ adapted endowment processes $\mathbf{w}$ (using Lemma B.2 below in the last step)

$$
\begin{aligned}
U^{\max }(\mathbf{w}) & =\frac{1}{1-\gamma} \sum_{t=0}^{T} e^{-\rho t} E\left[\mathrm{~cm}_{t}^{1-\gamma}\right] \\
& =\frac{1}{1-\gamma}\left(\sum_{t=0}^{T} E\left[\mathrm{~cm}_{t} M_{t}\right]\right)^{1-\gamma}\left(\sum_{t=0}^{T} e^{-\rho t / \gamma} E\left[M_{t}^{1-1 / \gamma}\right]\right)^{\gamma} \\
& =\frac{1}{1-\gamma}\left(\sum_{t=0}^{T} E\left[w_{t} M_{t}\right]\right)^{1-\gamma}\left(\sum_{t=0}^{T} e^{-\rho t / \gamma} E\left[M_{t}^{1-1 / \gamma}\right]\right)^{\gamma} .
\end{aligned}
$$


Using Lemma A.1 yields for any consumption stream $\mathbf{c}$

$$
\begin{aligned}
\frac{1}{1-\gamma} E\left[\sum_{t=0}^{T} e^{-\rho t} c_{t}^{1-\gamma}\right] & =\frac{1}{1-\gamma} E\left[\sum_{t=0}^{T} e^{-\rho t}\left(c_{t} M_{t}\right)^{1-\gamma} M_{t}^{-1+\gamma}\right] \\
& \leq \frac{1}{1-\gamma} \sum_{t=0}^{T} E\left[c_{t} M_{t}\right]^{1-\gamma}\left(e^{-\rho t / \gamma} E\left[M_{t}^{1-1 / \gamma}\right]\right)^{\gamma} \\
& \leq \frac{1}{1-\gamma}\left(\sum_{t=0}^{T} E\left[c_{t} M_{t}\right]\right)^{1-\gamma}\left(\sum_{t=0}^{T} e^{-\rho t / \gamma} E\left[M_{t}^{1-1 / \gamma}\right]\right)^{\gamma} .
\end{aligned}
$$

If we choose the optimal consumption stream c and use Lemma B.2 we obtain

$$
\begin{aligned}
U^{\max } & (\mathbf{w}+\Pi-\mathbf{Y}) \\
& \leq \frac{1}{1-\gamma}\left(\sum_{t=0}^{T} E\left[\left(w_{t}+\Pi_{t}-Y_{t}\right) M_{t}\right]\right)^{1-\gamma}\left(\sum_{t=0}^{T} e^{-\rho t / \gamma} E\left[M_{t}^{1-1 / \gamma}\right]\right)^{\gamma} .
\end{aligned}
$$

This together with (A.17) completes the claim.

Proof of Lemma 2.15. Suppose that there is an arbitrage opportunity. That is, there exist claims streams $\mathbf{Y}^{1}, \ldots, \mathbf{Y}^{k}$ and a portfolio strategy $\mathbf{x}$ such that

$$
-\sum_{i=1}^{k} \pi_{0}\left(\mathbf{Y}^{i}\right)+D_{\mathbf{x}, 0} \geq 0
$$

and

$$
\sum_{i=1}^{k} Y_{t}^{i}+D_{\mathbf{x}, t} \geq 0
$$

for all $t \geq 1$ and at least one of the inequalities is strict for some state. Then,

$$
\begin{aligned}
0<-\sum_{i=1}^{k} \pi_{0}\left(\mathbf{Y}^{i}\right) & +D_{\mathbf{x}, 0}+E\left[\sum_{t=1}^{T} M_{t}\left(\sum_{i=1}^{k} Y_{t}^{i}+D_{\mathbf{x}, t}\right)\right] \\
& =\sum_{i=1}^{k}\left(-\pi_{0}\left(\mathbf{Y}^{i}\right)+E\left[\sum_{t=1}^{T} M_{t} Y_{t}^{i}\right]\right) \leq 0,
\end{aligned}
$$

which is a contradiction.

\section{B. Proofs of Section 3}

In this section we prove our first two main results, the first order conditions for optimal consumption streams (Theorems 3.1 and 3.7). We start with the 
proofs of Lemmas 3.3-3.5. Moreover, we state two auxilary lemmas, Lemmas B.1 and B.2. These two lemmas are crucial for the understanding of the structure of optimal consumption stream when enlarging the underlying information from $\mathcal{F}$ to $\mathcal{G}$.

Proof of Lemma 3.3. For any asset $A_{j}$, the variable $M_{t+1}\left(q_{j, t+1}+D_{j, t+1}\right)$ is $\mathcal{F}_{t+1^{-}}$ measurable. Thus, under Assumption 3, $\mathbf{M}=\left(M_{t}\right)_{t}$ is also a state price density process for the market $(\mathcal{M}, \mathcal{G})$.

Proof of Lemma 3.4. The $\mathcal{H}_{t}$-measurability follows from the definition of $X_{t}$ and $\mathcal{H}_{t}$. The existence follows from the completeness of the market $(\mathcal{M}, \mathcal{F})$. Namely, by definition, any random variable $X_{t}$ measurable with respect to $\mathcal{H}_{t}=\sigma\left(\mathcal{F}_{t}, \mathcal{G}_{t-1}\right)$ can be represented as a sum $X_{t}=\sum_{i=1}^{K} Z_{i} V_{i}$, where $Z_{i}$ is $\mathcal{G}_{t-1^{-}}$ measurable and $V_{i}$ is $\mathcal{F}_{t}$ measurable for each $i$. On the other hand, since the market $(\mathcal{M}, \mathcal{F})$ is complete, there exist $\mathcal{F}_{t-1}$-measurable variables $Y_{i, j}$ such that $V_{i}=\sum_{j=1}^{L} Y_{i, j}\left(p_{j, t}+d_{j, t}\right)$ for each $i$. Consequently,

$$
X_{t}=\sum_{j=1}^{L}\left(\sum_{i=1}^{K} Z_{i} Y_{i, j}\right)\left(p_{j, t}+d_{j, t}\right)
$$

and, consequently, the required portfolio strategy is given by

$$
x_{j, t-1}=\sum_{i=1}^{K} Z_{i} Y_{i, j}
$$

for all assets $j=1, \ldots, L$. Identities (3.9)-(3.10) follow from Lemma 3.3.

Proof of Lemma 3.5. Let

$$
D_{\mathbf{x}, t}=X_{t}-E\left[\frac{M_{t+1}}{M_{t}} X_{t+1} \mid \mathcal{G}_{t}\right]
$$

be a dividend process of a portfolio strategy and $\left(X_{t}\right)_{t}$ the corresponding wealth process. $\left(X_{t}\right)_{t}$ is an arbitrary $\mathcal{H}$ adapted process. If $\mathbf{x}$ is an upper hedging strategy, $D_{\mathbf{x}, t}>Y_{t}$ for all $t \geq 1$. For $t=T$,

$$
X_{T} \geq Y_{T} \Leftrightarrow X_{T} \geq Y_{T}^{\text {sup }}
$$

and this is the exact lower bound. Suppose that we have proved that $X_{t+1} \geq$ $Y_{t+1}^{\text {sup }}$ and that this is the exact lower bound. Then,

$$
D_{\mathbf{x}, t} \geq Y_{t} \Leftrightarrow X_{t} \geq E\left[\frac{M_{t+1}}{M_{t}} X_{t+1} \mid G_{t}\right]+Y_{t} \geq Y_{t}+E\left[\frac{M_{t+1}}{M_{t}} Y_{t+1}^{\text {sup }} \mid G_{t}\right] .
$$


Since $X_{t}$ is $\mathcal{H}_{t}$-measurable, this implies

$$
X_{t} \geq Y_{t}^{\text {sup }}
$$

and, obviously, this is an exact lower bound. Continuing inductively, we get that the minimal number, sufficient to buy an upper hedging strategy, is $Y_{0}^{\text {sup }}$.

Lemma B.1. Suppose that Assumptions 1-3 are satisfied. The following are equivalent.

(a) A G adapted process $\left(R_{t}\right)_{t=0, \ldots, T}$ is a state density process for $(\mathscr{M}, \mathcal{G})$.

(b) For all $t=0, \ldots, T-1$

$$
E\left[R_{t+1} M_{t+1}^{-1} \mid \mathcal{H}_{t+1}\right]=R_{t} M_{t}^{-1} .
$$

(c) For all $\mathcal{G}$ adapted portfolio strategies $\mathbf{x}$

$$
\sum_{s=0}^{T} E\left[D_{\mathbf{x}, s} R_{s}\right]=0 .
$$

(d) There exist $Z_{1}, \ldots, Z_{T}$ with $Z_{t}$ is $\mathcal{G}_{t}$-measurable and $E\left[Z_{t} \mid \mathcal{H}_{t}\right]=0$ for all $t \geq 1$ such that

$$
R_{t} M_{t}^{-1}=1+Z_{1}+\ldots+Z_{t} .
$$

Proof of Lemma B.1. Proof $(b) \Rightarrow(a)$ Observe that we have

$$
\begin{aligned}
& E\left[\left(D_{j, t+1}+q_{j, t+1}\right) R_{t+1} \mid \mathcal{G}_{t}\right] \\
& \quad=E\left[\left(D_{j, t+1}+q_{j, t+1}\right) M_{t+1} E\left[R_{t+1} M_{t+1}^{-1} \mid \mathcal{H}_{t+1}\right] \mid \mathcal{G}_{t}\right] \\
& \quad=R_{t} M_{t}^{-1} E\left[\left(D_{j, t+1}+q_{j, t+1}\right) M_{t+1} \mid \mathcal{G}_{t}\right] .
\end{aligned}
$$

But then the claim follows from Assumption 3.

Proof $(a) \Rightarrow(b)$. Note that the state price density processes $\left(R_{t}\right)_{t}$ and $\left(M_{t}\right)_{t}$ satisfy (under Assumption 1 and in the view of the proof of Lemma 3.3)

$$
E\left[\left(D_{j, t+1}+q_{j, t+1}\right) M_{t+1}\left(R_{t+1} M_{t+1}^{-1}-R_{t} M_{t}^{-1}\right) \mid G_{t}\right]=0 .
$$

Observe that $\left(D_{j, t+1}+q_{j, t+1}\right) M_{t+1}$ is $\mathcal{F}_{t+1}$-measurable. Since we have assumed that the financial market $(\mathcal{M}, \mathcal{F})$ is complete we can replicate every $\mathcal{F}_{t+1}$-measurable claim $Y$ by the dividend process $\mathbf{D}_{\mathbf{x}}$ of an appropriate $\mathcal{F}$ adapted investment strategy $\mathbf{x}$. Hence, (B.7) implies that for every $\mathcal{F}_{t+1}$ measurable claim $Y$ 


$$
E\left[Y\left(R_{t+1} M_{t+1}^{-1}-R_{t} M_{t}^{-1}\right) \mid \mathcal{G}_{t}\right]=0
$$

Thus, $Y\left(R_{t+1} M_{t+1}^{-1}-R_{t} M_{t}^{-1}\right)$ is orthogonal to $\mathcal{G}_{t}$ which implies that for every $\mathcal{F}_{t+1}$ measurable random variable $Y$ and for every $\mathcal{G}_{t}$ measurable random variable $Z$ we have

$$
E\left[Y Z\left(R_{t+1} M_{t+1}^{-1}-R_{t} M_{t}^{-1}\right)\right]=0 .
$$

That is, $\left(R_{t+1} M_{t+1}^{-1}-R_{t} M_{t}^{-1}\right)$ is in the orthogonal complement of $\mathcal{H}_{t+1}$.

Proof $(a) \Rightarrow(c)$ is trivial whereas the Proof $(c) \Rightarrow(a)$ uses all strategies of the type $\mathbf{x}=\left(0, \ldots, 0, \mathbf{x}_{j}, 0, \ldots, 0\right)$ with $\mathbf{x}_{j}=\left(0, \ldots, 0, x_{j, t}, 0, \ldots, 0\right)$ and $x_{j, t} \mathcal{G}$ adapted. Proof $(b) \Leftrightarrow(d)$ is clear from the martingale assumption.

This completes the proof.

Lemma B.2. Assume Assumptions 1-3. The $\mathcal{G}$ adapted process $\mathbf{D}_{\mathbf{x}}$ is a dividend process if and only if

$$
\begin{aligned}
& E\left[\sum_{s=0}^{T} D_{\mathbf{x}, s} M_{s}\right]=0 \\
& E\left[\sum_{s=t}^{T} D_{\mathbf{x}, s} M_{s} \mid \mathcal{G}_{t}\right]=E\left[\sum_{s=t}^{T} D_{\mathbf{x}, s} M_{s} \mid \mathcal{H}_{t}\right] \text { for all } t=1, \ldots, T .
\end{aligned}
$$

Proof of Lemma B.2. Due to Lemma B.1 we know that there exists a state price density process if and only if there exists $Z_{0}=1, Z_{1}, \ldots, Z_{T}$ with $Z_{t} G_{t}$-measurable and $E\left[Z_{t} \mid \mathcal{H}_{t}\right]=0, t \geq 1$, such that

$$
\sum_{s=0}^{T} E\left[D_{\mathbf{x}, s} M_{s} \sum_{\tau=0}^{s} Z_{\tau}\right]=0 .
$$

The equality is equivalent to

$$
\sum_{\tau=0}^{T} E\left[\sum_{s=\tau}^{T} D_{\mathbf{x}, s} M_{s} Z_{\tau}\right]=0 .
$$

Proof "only if". If $\mathbf{D}_{\mathbf{x}}$ is a $\mathcal{G}$ adapted dividend process it satisfies (B.13) for all $Z_{0}=1, Z_{1}, \ldots, Z_{T}$ with $Z_{t} \mathcal{G}_{t}$ measurable and $E\left[Z_{t} \mid \mathcal{H}_{t}\right]=0, t \geq 1$. Hence, this implies that

$$
E\left[\sum_{s=0}^{T} D_{\mathbf{x}, s} M_{s} Z_{0}\right]=0 \text {, and }
$$




$$
E\left[\sum_{s=\tau}^{T} D_{\mathbf{x}, s} M_{s} \mid \mathcal{G}_{t}\right] \text { is orthogonal to all } Z_{\tau} \text { with } E\left[Z_{t} \mid \mathcal{H}_{t}\right]=0
$$

Hence (B.14) implies (B.10), and (B.15) implies that $E\left[\sum_{s=\tau}^{T} D_{\mathbf{x}, s} M_{s} \mid \mathcal{G}_{\tau}\right]$ is $\mathcal{H}_{\tau^{-}}$ measurable and which implies (B.11) due to $\mathcal{G}_{\tau} \supset \mathcal{H}_{\tau}$.

Proof "if". Choose $Z_{\tau} \mathcal{G}_{\tau}$-measurable with $E\left[Z_{t} \mid \mathcal{H}_{t}\right]=0$. Hence

$$
\begin{aligned}
E\left[\sum_{s=\tau}^{T} D_{\mathbf{x}, s} M_{s} Z_{\tau}\right] & =E\left[E\left[\sum_{s=\tau}^{T} D_{\mathbf{x}, s} M_{s} \mid \mathcal{G}_{t}\right] Z_{\tau}\right] \\
& =E\left[E\left[\sum_{s=\tau}^{T} D_{\mathbf{x}, s} M_{s} \mid \mathcal{H}_{t}\right] Z_{\tau}\right] \\
& =E\left[E\left[\sum_{s=\tau}^{T} D_{\mathbf{x}, s} M_{s} \mid \mathcal{H}_{t}\right] E\left[Z_{\tau} \mid \mathcal{H}_{t}\right]\right]=0 .
\end{aligned}
$$

This implies that $\mathbf{D}_{\mathbf{x}}$ is a dividend process because it is in the orthogonal complement of all $Z_{\tau}$ (which itself spans the orthogonal complement of $\mathcal{H}_{\tau}$ ).

Proof of Theorems 3.1 and 3.7. Note that Theorem 3.1 follows from Theorem 3.7 for $\mathcal{G}_{t}=\mathcal{F}_{t}$. Thus, it remains to prove Theorem 3.7.

Proof: first order conditions. For $t=0, \ldots, T-1$ we consider the portfolio positions $x_{t}=\left(x_{1, t}, \ldots, x_{L, t}\right)$ at time $t$ whose portfolio price at time $t$ is $\sum_{j=1}^{L} q_{j, t} x_{j, t}$. It gives wealth $X_{t+1}$ at time $t+1$. Now, we perturb one coordinate $\left(x_{1, t}, \ldots, x_{L, t}\right) \mapsto$ $\left(x_{1, t}, \ldots, x_{j-1, t}, x_{j, t}+\varepsilon, x_{j+1, t}, \ldots, x_{L, t}\right)$ of this portfolio. Observe that the perturbed portfolio satisfies

$$
\begin{aligned}
& \frac{\partial}{\partial \varepsilon}\left(\sum_{j=1}^{L} q_{j, t} x_{j, t}+q_{j, t} \varepsilon\right)=q_{j, t}, \\
& \frac{\partial}{\partial \varepsilon}\left(X_{t+1}+\left(q_{j, t+1}+D_{j, t+1}\right) \varepsilon\right)=q_{j, t+1}+D_{j, t+1} .
\end{aligned}
$$

Set $u(c)=c^{1-\gamma} /(1-\gamma)$. Hence, if the $\mathcal{G}$ adapted investment strategy $\mathbf{x}$ gives an optimal consumption stream, the derivative of the perturbed portfolio w.r.t. $\varepsilon$ needs to be 0 (relative to $\mathcal{G}_{t}$ ). This implies

$$
E\left[-e^{-\rho t} u^{\prime}\left(c_{t}\right) q_{j, t}+e^{-\rho(t+1)} u^{\prime}\left(c_{t+1}\right)\left(q_{j, t+1}+D_{j, t+1}\right) \mid \mathcal{G}_{t}\right]=0 .
$$

Hence, for all $j=1, \ldots, L$ and $t=0, \ldots, T-1$

$$
E\left[e^{-\rho(t+1)} u^{\prime}\left(c_{t+1}\right)\left(q_{j, t+1}+D_{j, t+1}\right) \mid \mathcal{G}_{t}\right]=e^{-\rho t} u^{\prime}\left(c_{t}\right) q_{j, t} .
$$


Thus, $R_{t}=e^{-\rho t} u^{\prime}\left(c_{t}\right)$ is a state price density process for the market $(\mathcal{M}, \mathcal{G})$. Applying Lemma B.1 completes the proof.

The proof is complete.

Proof of Theorem 3.8. We use the following well-known identity

$$
\lim _{\gamma \rightarrow+\infty}\left(E\left[X^{\gamma} \mid \mathcal{H}_{t}\right]\right)^{1 / \gamma}=\operatorname{esssup}\left[X \mid \mathcal{H}_{t}\right] .
$$

Using the first order conditions from Theorem 3.7 and sending $\gamma \rightarrow \infty$ we obtain in the limit

$$
\operatorname{essinf}\left[c_{t} \mid \mathcal{H}_{t}\right]=c_{t-1} \text {. }
$$

For $t=T$, this implies

$$
w_{T}-Y_{T}^{\text {sup }}+X_{T}=c_{T-1}=w_{T-1}-Y_{T-1}+X_{T-1}-M_{T-1}^{-1} E\left[M_{T} X_{T} \mid \mathcal{G}_{T-1}\right],
$$

and multiplying by $M_{T} M_{T-1}^{-1}$ and taking conditional expectation w.r.t. the measure $P\left[\cdot \mid \mathcal{G}_{T-1}\right]$, we get

$$
\begin{aligned}
& M_{T-1}^{-1} E[\left.M_{T} X_{T} \mid \mathcal{G}_{T-1}\right]=\frac{M_{T-1}}{M_{T-1}+E\left[M_{T} \mid \mathcal{G}_{T-1}\right]} \\
& \cdot E\left[\frac{M_{T}}{M_{T-1}}\left(w_{T-1}-Y_{T-1}+X_{T-1}+Y_{T}^{\text {sup }}-w_{T}\right) \mid \mathcal{G}_{T-1}\right] .
\end{aligned}
$$

Consequently,

$$
\begin{array}{r}
X_{T}=\frac{\left(w_{T-1}-Y_{T-1}+X_{T-1}\right)}{1+M_{T-1}^{-1} E\left[M_{T} \mid \mathcal{G}_{T-1}\right]}+Y_{T}^{\text {sup }}-w_{T} \\
-\frac{M_{T-1}^{-1} E\left[M_{T}\left(Y_{T}^{\text {sup }}-w_{T}\right) \mid \mathcal{G}_{T-1}\right]}{1+M_{T-1}^{-1} E\left[M_{T} \mid \mathcal{G}_{T-1}\right]},
\end{array}
$$

and

$$
\begin{aligned}
& c_{T-1}= \\
& \frac{X_{T-1}+w_{T-1}+M_{T-1}^{-1} E\left[M_{T} w_{T} \mid \mathcal{F}_{T-1}\right]-Y_{T-1}-M_{T-1}^{-1} E\left[M_{T} Y_{T}^{\text {sup }} \mid \mathcal{G}_{T-1}\right]}{1+M_{T-1}^{-1} E\left[M_{T} \mid \mathcal{G}_{T-1}\right]} .
\end{aligned}
$$

Proceeding inductively and using (B.22) in each step, it follows for all $t$

$$
c_{t}=\frac{X_{t}+M_{t}^{-1} E\left[\sum_{\tau=t}^{T} M_{\tau} w_{\tau} \mid \mathcal{F}_{t}\right]-Y_{t}-M_{t}^{-1} E\left[M_{t+1} Y_{t+1}^{\text {sup }} \mid \mathcal{G}_{t}\right]}{1+M_{t}^{-1} E\left[\sum_{\tau=t+1}^{T} M_{\tau} \mid \mathcal{G}_{t}\right]} .
$$


We rewrite the equation for the premium as follows, see (A.17), $\gamma>1$

$$
\begin{aligned}
& \left(-U^{\max }\left(\mathbf{w}+\Pi_{0}-\mathbf{Y}\right)\right)^{1 /(1-\gamma)} \\
& \quad=(\gamma-1)^{-1 /(1-\gamma)} E\left[\sum_{t=0}^{T} w_{t} M_{t}\right]\left(E\left[\sum_{t=1}^{T} e^{-t / \gamma} M_{t}^{1-1 / \gamma}\right]\right)^{\gamma /(1-\gamma)},
\end{aligned}
$$

and taking the limit $\gamma \rightarrow \infty$ we get

$$
\inf _{t} \operatorname{essinf}\left[c_{t}\right]=\frac{E\left[\sum_{t=0}^{T} w_{t} M_{t}\right]}{E\left[\sum_{t=0}^{T} M_{t}\right]} .
$$

But, by (B.22), we have inf $\operatorname{essinf}_{t}\left[c_{t}\right]=c_{0}$. Henceforth, by (B.27) and $M_{0}=1$, we have in the limiting case

$$
c_{0}=\frac{\pi_{0}(\infty)-Y_{0}^{\text {sup }}+E\left[\sum_{t=0}^{T} w_{t} M_{t}\right]}{E\left[\sum_{t=0}^{T} M_{t}\right]} .
$$

The proof is complete.

\section{Proofs of Section 4}

We define, iteratively, $w_{T+1}^{\inf }=0$ and a $\mathcal{H}$ adapted process $w_{t}^{\text {inf }}, t=1, \ldots, T+1$, by

$$
w_{t-1}^{\inf }=\operatorname{essinf}\left[w_{t-1}+E\left[\frac{M_{t}}{M_{t-1}} w_{t}^{\inf } \mid \mathcal{G}_{t-1}\right] \mid \mathcal{H}_{t-1}\right] .
$$

For complete markets, that is $\mathcal{F}_{t}=\mathcal{G}_{t}=\mathcal{H}_{t}$, we have

$$
w_{t}^{\mathrm{inf}}=E\left[\sum_{\tau=t}^{T} \frac{M_{\tau} w_{\tau}}{M_{t}} \mid \mathcal{G}_{t}\right] \text {. }
$$

The proof of Lemma 4.1 easily follows from the next Lemma.

Lemma C.1. The random functions defined in (4.1)-(4.3) exist if and only if $w_{0}^{\mathrm{inf}}>0$. Furthermore, $F_{t}$ is increasing, supported by the lower threshold $-w_{t-1}$ and

$$
\lim _{x \rightarrow w_{t-1}} F_{t}(x, \mathbf{w})=-w_{t}^{\inf } \text { and } \lim _{x \rightarrow \infty} F_{t}(x, \mathbf{w})=\infty,
$$


and $G_{t}$ is supported by the lower threshold $a_{t}=-w_{t-1}-E\left[\frac{M_{t}}{M_{t-1}} w_{t}^{\mathrm{inf}} \mid G_{t-1}\right]$, and

$$
\lim _{x \rightarrow a_{t}} G_{t}(x, \mathbf{w})=-E\left[\frac{M_{t}}{M_{t-1}} w_{t}^{\inf } \mid \mathcal{G}_{t-1}\right] \text { and } \lim _{x \rightarrow \infty} G_{t}(x, \mathbf{w})=\infty .
$$

In particular, in the complete market case the functions exist if and only if the intertemporal wealth $E\left[\sum_{t=0}^{T} M_{t} w_{t}\right]$ is positive.

Proof. We prove the statements inductively. We start with $t=T$. Observe that $G_{T+1}=0$ and construct $F_{T}$. It is clear that $-w_{T-1}$ is the supporting lower threshold for $F_{T}$ (otherwise the right-hand side of (4.1) is not well-defined) and from the left-hand side of (4.1) we obtain

$$
\lim _{x \rightarrow w_{T-1}} F_{T}(x, \mathbf{w})=-w_{T}^{\text {inf }} \quad \text { and } \quad \lim _{x \rightarrow \infty} F_{T}(x, \mathbf{w})=\infty .
$$

Since the probability space is finite (Technical Assumption 1), the left-hand side of (4.1) blows up as $F_{T}(x, \mathbf{w}) \rightarrow-w_{T}^{\text {inf }}$. Hence, existence, uniqueness and monotonicity of $F_{T}$ are clear.

We now assume that $F_{t}$, as stated, exists (we have initialized the induction for $t=T$ ) and prove that then $G_{t}$ can be constructed. Consider

$$
y-E\left[\frac{M_{t}}{M_{t-1}} F_{t}(x-y, \mathbf{w}) \mid \mathcal{G}_{t-1}\right]=0 .
$$

The left hand-side is defined and monotone increasing in $y$ on the interval $\left(-\infty, x+w_{t-1}\right)$ (since $F_{t}$ is monoton increasing). Moreover,

$$
\begin{aligned}
-\infty & =\lim _{y \rightarrow-\infty}\left(y-E\left[\frac{M_{t}}{M_{t-1}} F_{t}(x-y, \mathbf{w}) \mid \mathcal{G}_{t-1}\right]\right) \\
& <\lim _{y \rightarrow-w_{t-1}}\left(y-E\left[\frac{M_{t}}{M_{t-1}} F_{t}(x-y, \mathbf{w}) \mid \mathcal{G}_{t-1}\right]\right) \\
& =x+w_{t-1}+E\left[\frac{M_{t}}{M_{t-1}} w_{t}^{\inf } \mid \mathcal{G}_{t-1}\right],
\end{aligned}
$$

and therefore $G_{t}$ is defined and monotone increasing in $x$ on the interval $\left(-w_{t-1}-E\left[\frac{M_{t}}{M_{t-1}} w_{t}^{\mathrm{inf}} \mid \mathcal{G}_{t-1}\right], \infty\right)$. Note that by definition

$$
G_{t}(x, \mathbf{w})-E\left[\frac{M_{t}}{M_{t-1}} F_{t}\left(x-G_{t}(x, \mathbf{w}), \mathbf{w}\right) \mid G_{t-1}\right]=0 .
$$


Hence, since the first term on the left-hand side of (C.8) is increasing, also $x-G_{t}(x, \mathbf{w})$ needs to be a monotone increasing function in $x$ for any $t$ (since by induction assumption $F_{t}$ is increasing).

But then, for $t<T, F_{t}$ can easily be constructed inductively from (4.1) and the fact that $x-G_{t+1}(x, \mathbf{w})$ is a monotone increasing function. Moreover, $F_{t}$ satisfies all the required assumptions.

Observe that from $X_{0}=0$ follows that the inductive recursion can only be started if $0 \in\left(-w_{0}-E\left[M_{1} w_{1}^{\text {inf }} \mid \mathcal{G}_{0}\right], \infty\right)$ which is equivalent to $w_{0}^{\text {inf }}>0$.

Proof of Theorem 4.3. Note that the convention $X_{0}=0$ needs always to hold true since we start to invest at time $t=0$, i.e. $x_{j-1} \equiv 0$.

The optimal consumption stream satisfies the first order conditions (3.16). Hence, using (3.15) and (3.16), $\left(X_{t}\right)_{t}$ is an optimal wealth process only if the following identity holds

$$
\begin{array}{r}
e^{-\rho} E\left[\left(w_{t}+X_{t}-E\left[\frac{X_{t+1} M_{t+1}}{M_{t}} \mid \mathcal{G}_{t}\right]\right)^{-\gamma} M_{t}^{-1} \mid \mathcal{H}_{t}\right] \\
=\left(w_{t-1}+X_{t-1}-E\left[\frac{X_{t} M_{t}}{M_{t-1}} \mid \mathcal{G}_{t-1}\right]\right)^{-\gamma} M_{t-1}^{-1} .
\end{array}
$$

Therefore we need to prove that (C.9) holds true for $\left(X_{t}\right)_{t}$ defined by (4.4).

Using (4.4) and (4.3) we have, for all $t=1, \ldots, T$,

$$
X_{t}=H_{t}\left(X_{t-1}, \mathbf{w}\right)=F_{t}\left(X_{t-1}-G_{t}\left(X_{t-1}, \mathbf{w}\right), \mathbf{w}\right) .
$$

Note that $X_{T+1}=0$, due to $x_{j, T}=0$ for all $j=1, \ldots, L$. Set $H_{T+1} \equiv F_{T+1} \equiv$ $G_{T+1} \equiv 0$. Then the left-hand side of (C.9) can be rewritten as follows for $t=1, \ldots, T$ (using (C.10))

$$
\begin{aligned}
e^{-\rho} E\left[\left(w_{t}+H_{t}\left(X_{t-1}, \mathbf{w}\right)-E\left[\frac{H_{t+1}\left(X_{t}, \mathbf{w}\right) M_{t+1}}{M_{t}} \mid \mathcal{G}_{t}\right]\right)^{-\gamma} M_{t}^{-1} \mid \mathcal{H}_{t}\right] \\
=e^{-\rho} E\left[\left(w_{t}+F_{t}\left(X_{t-1}-G_{t}\left(X_{t-1}, \mathbf{w}\right), \mathbf{w}\right)\right.\right. \\
\left.\left.\quad-E\left[\frac{M_{t+1}}{M_{t}} F_{t+1}\left(X_{t}-G_{t+1}\left(X_{t}, \mathbf{w}\right), \mathbf{w}\right) \mid \mathcal{G}_{t}\right]\right)^{-\gamma} M_{t}^{-1} \mid \mathcal{H}_{t}\right] .
\end{aligned}
$$

Observe that $X_{t}$ is $\mathcal{H}_{t}$-measurable (see Lemma 3.4) and hence $\mathcal{G}_{t}$-measurable. Hence, using (4.2), we find

$$
\begin{gathered}
E\left[\frac{M_{t+1}}{M_{t}} F_{t+1}\left(X_{t}-G_{t+1}\left(X_{t}, \mathbf{w}\right), \mathbf{w}\right) \mid \mathcal{G}_{t}\right]=G_{t+1}\left(X_{t}, \mathbf{w}\right) \\
=G_{t+1}\left(F_{t}\left(X_{t-1}-G_{t}\left(X_{t-1}, \mathbf{w}\right), \mathbf{w}\right), \mathbf{w}\right) .
\end{gathered}
$$


The left-hand side of (C.9) is equal to (using (4.1) and the fact that $X_{t-1}-$ $G_{t}\left(X_{t-1}, \mathbf{w}\right)$ is $\mathcal{H}_{t}$-measurable)

$$
\begin{aligned}
e^{-\rho} E & {\left[\left(w_{t}+F_{t}\left(X_{t-1}-G_{t}\left(X_{t-1}, \mathbf{w}\right), \mathbf{w}\right)-G_{t+1}\left(X_{t}, \mathbf{w}\right)\right)^{-\gamma} M_{t}^{-1} \mid \mathcal{H}_{t}\right] } \\
& =\left(w_{t-1}+X_{t-1}-G_{t}\left(X_{t-1}, \mathbf{w}\right)\right)^{-\gamma} M_{t-1}^{-1} .
\end{aligned}
$$

In view of (4.2) and (C.10) this last expression is equal to the right-hand side of (C.9). This completes the proof.

\section{Proofs of Section 5}

Proof of Proposition 5.5. Observe that if we have borrowing constraints, we have less admissible $\mathcal{G}$ adapted trading strategies $\mathbf{x}$. Hence, the utility of the optimal consumption stream becomes smaller under borrowing constraints, which implies that (using monotonicity) both $\pi_{0}^{a}$ and $\pi_{a n n}^{a}$ are monotone decreasing with $a$.

Now, let us prove convexity of $\pi_{a n n}^{a}$. The convexity of $\pi_{0}^{a}$ follows analogously. Let $\mathbf{x}^{a}$ be the optimal portfolio strategy under the borrowing constraint $a$ and let $\mathbf{x}^{b}$ be the optimal portfolio strategy under the constraint $b$. Then, obviously, $\frac{1}{2}\left(\mathbf{x}^{a}+\mathbf{x}^{b}\right)$ satisfies the borrowing constraint determined by $\frac{1}{2}(a+b)$. Then, using the concavity of $u(c)=c^{1-\gamma} /(1-\gamma)$,

$$
\begin{aligned}
U^{\max }(\mathbf{w}) & =\frac{1}{2}\left(U\left(\mathbf{w}-\mathbf{Y}+\Pi_{a n n}^{a}+\mathbf{D}_{\mathbf{x}^{a}}\right)+U\left(\mathbf{w}-\mathbf{Y}+\Pi_{a n n}^{b}+\mathbf{D}_{\mathbf{x}^{b}}\right)\right) \\
& \leq U\left(\frac{1}{2}\left(\mathbf{w}-\mathbf{Y}+\Pi_{a n n}^{a}+\Pi_{a n n}^{b}\right)+\mathbf{D}_{\frac{1}{2}\left(\mathbf{x}^{a}+\mathbf{x}^{b}\right)}\right) \\
& \leq U^{\max , \frac{1}{2}(a+b)}\left(\mathbf{w}-\mathbf{Y}+\frac{1}{2}\left(\Pi_{a n n}^{a}+\Pi_{a n n}^{b}\right)\right)
\end{aligned}
$$

and, consequently

$$
\frac{1}{2}\left(\Pi_{a n n}^{a}+\Pi_{a n n}^{b}\right) \geq \Pi_{a n n}^{\frac{1}{2}(a+b)} .
$$

Finally, we prove the inequality

$$
\pi_{0}^{a} \leq \tilde{\pi}_{0}^{a} \stackrel{\text { def }}{=} \pi_{a n n}^{a} \sum_{t=1}^{T} E\left[M_{t}\right]
$$

We show that for every $\mathcal{G}$ adapted trading strategy $\mathbf{x}$ for the premium flow $\Pi_{a n n}^{a}$ there exists a $\mathcal{G}$ adapted trading strategy $\tilde{\mathbf{x}}$ for the static premium $\tilde{\Pi}_{0}^{a}=$ $\left(\tilde{\pi}_{0}^{a}, 0, \ldots, 0\right)$ that generates the same consumption stream. Then, clearly,

$$
U^{\max , a}\left(\tilde{\Pi}_{0}^{a}+\mathbf{w}-\mathbf{Y}\right) \geq U^{\max , a}\left(\Pi_{a n n}^{a}+\mathbf{w}-\mathbf{Y}\right)=U^{\max }(\mathbf{w}),
$$


and therefore $\Pi_{0}^{a} \leq \tilde{\Pi}_{0}^{a}$.

If $\left(X_{t}\right)_{t}$ denotes the wealth process of the trading strategy $\mathbf{x}$ then we define the wealth process

$$
\tilde{X}_{t}=X_{t}+\pi_{a n n}^{a} M_{t}^{-1} E\left[\sum_{s=t}^{T} M_{s} \mid \mathcal{F}_{t}\right]
$$

Obviously, if $X_{t}$ satisfies the borrowing constraints, then so does $\tilde{X}_{t}$. Furthermore, if the consumption stream $\tilde{\mathbf{c}}$ for $\tilde{\pi}_{0}^{a}$ and $\tilde{X}_{t}$ is given by

$$
\tilde{c}_{0}=w_{0}+\tilde{\pi}_{0}^{a}-E\left[M_{1} \tilde{X}_{1}\right],
$$

and for $t \geq 1$

$$
\tilde{c}_{t}=w_{t}-Y_{t}+\tilde{X}_{t}-M_{t}^{-1} E\left[M_{t+1} \tilde{X}_{t+1} \mid \mathcal{G}_{t}\right]
$$

then we can write

$$
\tilde{c}_{t}=\pi_{a n n}^{a}+w_{t}-Y_{t}+X_{t}-M_{t}^{-1} E\left[M_{t+1} X_{t+1} \mid \mathcal{G}_{t}\right]=c_{t} .
$$

This completes the proof.

Proof of Theorem 5.6. The proof is an application of the standard Kuhn-Tucker theorem (see e.g. Ingersoll [20]). We consider the consumption stream $\mathbf{c}$ as a function of the investment strategy $\mathbf{x}$ for a fixed time point $t=0, \ldots, T-1$. This means that we want to maximize over the $G_{t}$-measurable investments $x_{t}=$ $\left(x_{j, t}\right)_{j=1, \ldots, L}$. Henceforth, we consider

$$
X_{t+1}=X_{t+1}\left(x_{t}\right)=\sum_{j=1}^{L}\left(D_{j, t+1}+q_{j, t+1}\right) x_{j, t} .
$$

We define

$$
\begin{aligned}
& f\left(x_{t}\right)=\frac{c_{t}^{1-\gamma}\left(x_{t}\right)}{1-\gamma}+e^{-\rho} E\left[\frac{c_{t+1}^{1-\gamma}\left(x_{t}\right)}{1-\gamma} \mid \mathcal{G}_{t}\right], \\
& g\left(x_{t}\right)=E\left[\frac{M_{t+1} X_{t+1}\left(x_{t}\right)}{M_{t}} \mid \mathcal{G}_{t}\right],
\end{aligned}
$$

$f\left(x_{t}\right)$ describes all terms of the consumption stream $\mathbf{c}$ that contain the investments $x_{t}$ and $g\left(x_{t}\right) \geq-a$ describes the borrowing constraint. The Kuhn-Tucker theorem gives (5.12) and for all $j=1, \ldots, L$

$$
\begin{aligned}
0 \leq & \lambda_{t}\left(\operatorname{grad} g\left(x_{t}\right)\right)_{j}=\lambda_{t} q_{j, t} \\
& =\left(\operatorname{grad} f\left(x_{t}\right)\right)_{j}=-c_{t}^{-\gamma} q_{j, t}+e^{-\rho} E\left[c_{t+1}^{-\gamma}\left(D_{j, t+1}+q_{j, t+1}\right) \mid \mathcal{G}_{t}\right] .
\end{aligned}
$$


This now implies (5.11) (where the proof is completely analogous to the proof (a) $\Rightarrow$ (b) in Lemma B.1), namely $e^{-p} c_{t+1}^{-\gamma} M_{t+1}^{-1}-\left(\lambda_{t}-c_{t}^{-\gamma}\right) M_{t}^{-1}$ is in the orthogonal complement of $\mathcal{H}_{t+1}$. This completes the proof.

Proof of Lemma 5.3. We prove the claim by the backward induction. Note that $X_{T}$ must satisfy

$$
X_{T}>\left(-w_{T}\right)^{\text {sup }, a}=\left(-w_{T}\right)^{\text {sup }},
$$

and any $X_{T}$ satisfying (D.14) generates positive consumption. Now, suppose we have proved this for $t+1$ and let us prove it for $t$. We have

$$
c_{t}=w_{t}+X_{t}-E\left[\frac{M_{t+1}}{M_{t}} X_{t+1} \mid \mathcal{G}_{t}\right] .
$$

From the induction assumption we know that $X_{t+1} \geq\left(-w_{t+1}\right)^{\text {sup, } a}$ and any $X_{t+1}$ satisfying this inequality generates a positive consumption. But, because of the borrowing constraints, we must also have

$$
E\left[\frac{M_{t+1}}{M_{t}} X_{t+1} \mid \mathcal{G}_{t}\right] \geq-a .
$$

Thus, any $X_{t}$ satisfying

$$
X_{t}>-w_{t}+\max \left\{E\left[\frac{M_{t+1}}{M_{t}}\left(-w_{t+1}\right)^{\text {sup }, a} \mid \mathcal{G}_{t}\right],-a\right\}
$$

will generate a positive consumption. Now, since $X_{t}$ is $\mathcal{H}_{t}$-measurable, we take $\mathcal{H}_{t}$-conditional supremum to get the exact upper bound. The proof is complete.

Proof of Lemma 5.7. The fact that the functions exist follows simply from the Kuhn-Tucker Theorem (see Ingersoll [20] and proof of Theorem 5.6): we are maximizing a strictly concave function on a convex set and therefore the maximum is achieved in a point satisfying the Kuhn-Tucker conditions. The only point we must be careful in is that the maximum is not achieved at a point where $c_{t}=0$. But, this is impossible because the CRRA utility function satifies the standard Inada conditions:

$$
\lim _{c \rightarrow 0} u^{\prime}(c)=\lim _{c \rightarrow 0} c^{-\gamma}=+\infty .
$$

This argument gives abstract existence of the unique solution to the equations but it is not constructive. It is possible to give a constructive argument, completely similar to the case with no constraints (see proof of Lemma C.1). 
The only part that is slightly different, is the construction of the $\Lambda$-function. This is explained. Since $F_{t}$ is monotone increasing in $x$, it follows from (5.14) that $\tilde{G}_{t}^{(1)} \leq-a$ if and only if

$$
E\left[\frac{M_{t}}{M_{t-1}} F_{t}(x+a, 0, \mathbf{w}) \mid \mathcal{G}_{t-1}\right] \leq-a .
$$

Now, it follows from the definition that $F_{t}$ is monotone increasing in $\lambda$ and

$$
\lim _{\lambda \uparrow c_{t}^{-\gamma}} F(x, \lambda, \mathbf{w})=\infty .
$$

Therefore, by continuity, there exists a unique $\lambda$ solving

$$
E\left[\frac{M_{t}}{M_{t-1}} F_{t}(x+a, \lambda, \mathbf{w}) \mid \mathcal{G}_{t-1}\right]=-a .
$$

This completes the proof.

Proof of Theorem 5.8.

The proof is completely analogous to the proof of Theorem 4.3.

\section{ACKNOWLEDGEMENT}

We thank anonymous referees who gave several useful comments on earlier versions of this paper which led to a substantial improvement.

\section{REFERENCES}

[1] Barrieu, P. and El Karoui, N. (2007) Pricing, hedging and optimal designing derivatives via minimization of risk measures. To appear in: Volume on Indifference Pricing, R. Carmona (Ed.), Princeton University Press.

[2] Bühlmann, H., Delbaen, F., Embrechts, P. and Shiryaev, A.N. (1998) On the Esscher transform in discrete finance models, Astin Bulletin 28, 171-186.

[3] Cochrane, J.H. (2001) Asset Pricing. Princeton University Press, Princeton and Oxford.

[4] Davis, M.H.A. (1998) Option pricing in incomplete markets. In: Mathematics of Derivative Securities, M.A.H. Dempster and S.R. Pliska (Eds.), Cambridge University Press.

[5] De FinetTi, B. (1957) Su un'impostazion alternativa dell teoria collectiva dell richio. Transactions of the XVth international congress of Actuaries 2, 433-443.

[6] De Jong, F. (2005) Valuation of pension liabilities in incomplete markets. Preprint, DNB Working Paper 67, December 2005, Netherlands.

[7] Delbaen, F., Grandits, P., Rheinlander, T., Samperi, D., Schweizer, M. and Stricker, C. (2002) Exponential hedging and entropic penalties. Math. Finance 12, 99-123.

[8] Delbaen, F. and Schachermayer, W. (1994) A general version of the fundamental theorem of asset pricing. Mathematische Annalen 300, 463-520.

[9] Dickson, D.C.M. and Waters, H.R. (2003) Some optimal dividends problems. Astin Bulletin 34, 49-74. 
[10] Duffie, D. (1996) Dynamic Asset Pricing Theory. 2nd Edition. Princeton University Press.

[11] ElliotT, R.J. and KopP, P.E. (2005) Mathematics of Financial Markets. 2nd Edition. Springer Finance, NY.

[12] FILIPOVIC, D. and ZABCZYK, J. (2002) Markovian term structure models in discrete time. Ann. Applied Prob. 12(2), 710-729.

[13] Föllmer, H. and Schied, A. (2004) Stochastic Finance: An Introduction in Discrete Time. 2nd Edition. De Gruyter Studies in Mathematics 27, Berlin.

[14] He, H. and Pearson, N.D. (1991) Consumption and portfolio policies with incomplete markets and short-sale constraints: the finite-dimensional case. Math. Finance 1(3), 1-10.

[15] Henderson, V. (2002) Valuation of claims on nontraded assets using utility maximation. Math. Finance 12, 351-373.

[16] Henderson, V. (2005) Explicit solutions to an optimal portfolio choice problem with stochastic income. J. Economic Dynamics and Control 29, 1237-1266.

[17] Henderson, V. and Hobson, D. (2007) Utility indifference pricing - an overview. To appear in: Volume on Indifference Pricing, R. Carmona (Ed.), Princeton University Press.

[18] Hodges, S.D. and Neuberger, A. (1989) Optimal replication of contingent claims under transaction costs. Review of Futures Markets 8, 222-239.

[19] Horst, U. and Muller, M. (2007) On the spanning property of risk bonds priced by equilibrium. To appear in Mathematics of Operations Research.

[20] Ingersoll, J.E. (1987) Theory of Financial Decision Making. Rowman and Littlefield Publishers, Maryland.

[21] KaratZas, I. and Zitkovic, G. (2003) Optimal consumption from investment and random endowment in incomplete semimartingale markets, Ann. Prob. 31(4), 1821-1858.

[22] Malamud, S. and Trubowitz, E. (2006) A unified approach to market incompleteness. Preprint, SSRN, http://papers.ssrn.com/sol3/papers.cfm?abstract_id $=990328$.

[23] Malamud, S. and Trubowitz, E. (2006) Asset pricing for idiosyncratically incomplete markets. Preprint, SSRN, http://papers.ssrn.com/sol3/papers.cfm?abstract_id $=912788$.

[24] Merton, R.C. (1971) Optimum consumption and portfolio rules in a continuous-time model. J. Economic Theory 3(4), 373-413.

[25] Muermann, A. (2003) Actuarially consistent valuation of catastrophe derivatives. Wharton Financial Institution Center, working paper 03-18.

[26] Musiela, M. and Zariphopoulou, T. (2004) An example of indifference prices under exponential preferences. Finance Stochast. 8, 229-239.

[27] Musiela, M. and Zariphopoulou, T. (2004) A valuation algorithm for indifference prices in incomplete markets. Finance Stochast. 8, 399-414.

[28] Pelsser, A. (2006) Pricing insurance contracts: an incomplete market approach. Notes ACFI seminar, 2 November 2006, University of Amsterdam.

[29] Rouge, R. and El Karoui, N. (2000) Pricing via utility maximization and entropy. Math. Finance 10, 259-276.

[30] Schachermayer, W. (1999) Utility Maximisation in Incomplete Markets. In: Stochastic Methods in Finance, M. Fritelli and W. Runggaldier (Eds.), Springer Lecture Notes in Mathematics 1856, 225-288.

[31] Sснмоск, U. (1999) Estimating the value of the WinCAT coupons of the Winterthur Insurance Convertible Bond: a study of model risk. Astin Bulletin 29, 101-163.

[32] Tsanakas, A. and Christofides, N. (2006) Risk exchange with distorted probabilities. Astin Bulletin 36, 219-244.

[33] Wüthrich, M.V., Bühlmann, H. and Furrer, H. (2008) Market-Consistent Actuarial Valuation. EAA Lecture Notes, Springer.

MARIO V. WÜTHRICH

ETH Zurich, Department of Mathematics

CH-8092 Zurich

Switzerland 